\title{
Piecemeal regulation of convergent neuronal lineages by bHLH transcription factors in C. elegans
}

\author{
Neda Masoudi ${ }^{1}$, Eviatar Yemini ${ }^{1}$, Ralf Schnabel ${ }^{2}$ and Oliver Hobert ${ }^{1}$ \\ ${ }^{1}$ Department of Biological Sciences, Columbia University, Howard Hughes Medical \\ Institute, New York, USA \\ ${ }^{2}$ Institute of Genetics, Technische Universität Braunschweig, Germany
}

\begin{abstract}
Classic cell lineage studies in the nematode Caenorhabditis elegans as well as recent lineage tracing in vertebrates have shown that cells of the same type can be generated by distinct cellular lineages that originate in different parts of the developing embryo ("lineage convergence"). Several C. elegans neuron classes composed of left/right or radially symmetric class members display such lineage convergence, in that individual neurons of the same class derive from distinct, non-bilaterally symmetric lineages. We show here that the $C$. elegans Atonal homolog lin-32/Ato, a bHLH transcription factor, is differentially expressed in neuronal lineages that give rise to left/right or radially symmetric class members. Loss of lin-32/Ato results in the selective loss of the expression of panneuronal markers and terminal selector-type transcription factors that confer neuron class-specific features. We discovered that another bHLH transcription factor, the Achaete Scute-homolog $h / h-14$ is expressed in mirror image pattern to lin$32 /$ Ato in a subset of the left/right symmetric neuron pairs and is required to induce neuronal identity and terminal selector expression on the contralateral side of the animal. These findings demonstrate that distinct lineage histories converge via distinct bHLH factors on the level of induction of terminal selector identity determinants, which thus serve as integrators of distinct lineage histories. We also describe neuron-toneuron identity transformations in lin-32/Ato mutants, which we propose to also be the result of misregulation of terminal selector gene expression.
\end{abstract}




\section{INTRODUCTION}

The completely elucidated lineage history of every individual cell in the nematode C. elegans provides an excellent opportunity to study how lineage affects cellular identity (SULSTON AND HORVITZ 1977; SULSTON et al. 1983). One intriguing revelation of the lineage description is that phenotypically similar cells can have different lineage histories. This is particularly evident in the nervous system, composed of 302 neurons in the hermaphrodite. Based on anatomy, function and molecular profiles, these 302 neurons can be grouped into 118 different classes, with members of each class being phenotypically similar and often completely indistinguishable by any known criterion (WHITE et al. 1986; HOBERT et al. 2016; TAYLOR et al. 2020). Members of individual neuron classes can have very similar lineage histories. For example, many ventral cord motor neuron classes are composed of members with similar lineage histories (SULSTON et al. 1983). Also, many classes of bilaterally symmetric neuron pairs are composed of two members whose developmental history is very similar (SULSTON et al. 1983). However, member of the same neuron class can also very distinct lineage histories. For example, the four bilaterally symmetric cephalic CEP sensory neurons, composed of a bilaterally symmetric ventral neuron pair (CEPV left and CEPV right) and a bilaterally symmetric dorsal neuron pair (CEPD left and right) share similar overall morphology, similar patterns of synaptic connectivity (WHITE et al. 1986) and similar molecular composition (TAYLOR et al. 2020). However, the CEPD pair and CEPV pair come from different neuroblast lineages (SULSTON et al. 1983). Members of other radiallysymmetric neuron classes are also phenotypically indistinguishable, despite distinct lineage histories; for example, the IL1, IL2, and OLQ neuron classes are composed of ventral and dorsal pairs which each have distinct lineage histories (SULSTON et al. 1983). Even neuron classes that are only composed of two bilaterally symmetric can be defined by two lineally distinct neurons. For example, the left and right ASE neuron derive from different embryonic blastomere, $A B a$ and ABp (SULSTON et al. 1983). Thus, distinct lineage histories can converge onto similar neuronal identities. Similar lineage convergence phenomena have recently been observed in vertebrates as well, including vertebrates (MCKENNA et al. 2016; WAGNER et al. 2018; CAO et al. 2019; CHAN et al. 
2019). For example, specific types of excitatory and inhibitory neurons of the mouse CNS develop through multiple, convergent trajectories(CAO et al. 2019).

How is such convergence achieved? Based on past studies of neuronal identity control, one point of convergence of distinct lineage histories are terminal selector transcription factors - post-mitotically expressed master regulators of neuron identity (HOBERT 2016). For example, the two lineally distinct ASE neurons both eventually turn on the terminal selector CHE-1, which instructs ASE neuron identity. Similarly, all six members of the IL2 neuron class, despite their distinct lineage histories, co-express the terminal selectors unc-86, sox-2 and cfi-1, which cooperate to control the expression of IL2 identity features (SHAHAM AND BARGMANN 2002; ZHANG et al. 2014; VIDAL et al. 2015). Similarly, the left and right CAN neurons, which display non-symmetric, distinct lineage histories, coexpress the ceh-10 homeobox gene, required to specify the identity of both of these neurons (FORRESTER et al. 1998; WENICK AND HOBERT 2004). These observations indicate that different lineage histories converge onto the expression of similar terminal selectors and that terminal selectors are therefore integrators of distinct lineage histories. What then are the molecular factors that converge in a lineagespecific manner to drive individual terminal selectors in a neuron type-specific manner?

Recent scRNA analysis of developing embryos has revealed molecular correlates to distinct lineage histories, such that the precursors of cells with similar terminal identities were shown to display distinct gene expression profiles, exactly as predicted by their distinct lineage histories (SULSTON et al. 1983; PACKER et al. 2019). However, no specific genetic factors have been shown so far to differentially control the identity of cells that converge on similar cell-fate outcomes. In this paper, we show that the C. elegans Atonal homolog lin-32/Ato (ZHAO AND EMMONS 1995) is differentially expressed in distinct lineages of cells that phenotypically converge on the same neuronal cell classes and, henceforth, selectively affects the specification of only some class members. Moreover, we discovered that the Achaete Scute homolog hlh-14 (FRANK et al. 2003) displays the mirror image expression to lin-32/Ato and functions on the contralateral side of the animal. 
Our analysis of bHLH transcription factors was originally motivated by our quest to understand how the expression of terminal selector transcription factors is controlled. Terminal selectors have emerged as key regulators of neuronal identity throughout the entire nervous system (HOBERT 2016), yet we understand little about how their expression is induced in the embryo (BERTRAND AND HOBERT 2009; MuRGAN et al. 2015). Based on a previously reported effect of lin-32/Ato on the expression of the terminal selectors unc-86 (BAUMEISTER et al. 1996) and mec-3 (Way et al., 1992), we sought to investigate the role of lin-32/Ato further. In a number of different organisms, from flies to worms to vertebrates, Atonal orthologs have mostly been characterized for the proneural activity that imposes neuronal identity on neuroectodermal progenitor cells (BERTRAND et al. 2002; BAKER AND BROWN 2018). Loss of such proneural activity results in a conversion from a neuronal to an ectodermal skin cell fate. Such proneural functions have been defined for lin-32/Ato in the context of number of peripheral sense organs in $C$. elegans, including the postdeirid lineage, the $Q$ lineage, and ray lineages in the male tail (ChAlfie AND Au 1989; ZhAO AND EMmONS 1995; PORTMAN AND EMmONS 2000; ZHU et al. 2014). These proneural functions are evidenced by lineage changes in which neuroblasts that normally divide to generate multiple distinct neuron types instead convert to skin cells, leading to a failure to generate a number of neuron types (CHALFIE AND Au 1989; ZHAO AND EMMONS 1995; PORTMAN AND EMMONS 2000; ZHU et al. 2014). However, other effects on neural lineages have been observed in lin-32/Ato mutants as well. For example, after its proneural role early in lineage development, lin-32/Ato has also been implicated in controlling later aspects of neuronal differentiation in the $Q$ and ray lineages (PORTMAN AND EMmONs 2000; MILLER AND PORTMAN 2011; ZHU et al. 2014). Cell identity fate transformations are also evident in a subset of dopaminergic neuronproducing lineages (DOITSIDOU et al. 2008) as well as in glia cells (ZHANG et al. 2020). lin-32/Ato has also been shown to affect the differentiation and/or function of a number of additional neuron types, including oxygen sensory neurons (RoJo RoMANOs et al. 2017), touch receptor neurons (MITANI et al. 1993), anterior ganglion neurons (BAUMEISTER et al. 1996; SHAHAM AND BARGMANN 2002), and the AIB interneuron (HoRI et al. 2018). However, it was left unclear whether these defects are proneural lineage defects, cell-identity transformation defects, or some combination of both these effects. 
Here, to facilitate a comprehensive analysis of lin-32/Ato function, we begin by describing the expression pattern of gfp-tagged LIN-32/Ato. While the expression of lin$32:: g f p$ is consistent with a number of previously described functions of lin-32/Ato, we identified sites of expression that led us to explore novel functions of lin-32/Ato. One consistent theme of lin-32/Ato function is its effect on the expression of terminal selector-type transcription factors. Our findings for lin-32, as well as another bHLH family of the Atonal superfamily, $h / h-14$, provide novel insights into the molecular basis for the convergence of distinct lineage history onto similar cellular differentiation events, via the regulation of terminal selector expression. 


\section{MATERIAL AND METHODS}

\section{Strains}

Strains were maintained by standard methods (BRENNER 1974). Previously described strains used in this study are as follow.

Mutant alleles lin-32(tm1446) (DoITsidou et al. 2008), hlh-14(tm295) (PoOLE et al. 2011).

Reporter alleles: lin-11(ot958[lin-11::gfp::FLAG]) (REILLY et al. 2020), ceh32(ot1040[ceh-32::gfp]) (kindly provided by Cyril Cros), unc-86(ot879[unc86::nNeonGreen]) (SERRANO-SAIZ et al. 2018), unc-42(ot986[unc-42::gfp])(BERGHOFF et al. 2021).

Reporter transgenes: nls394 (ngn-1::gfp) (NAKANO et al. 2010), otls339 (ceh43 fosmid.::gfp) (DoITsIDOU et al. 2013), otls703(Is[flp-3::mCherry]); myls13 (Is[klp-6::gfp]) (kindly provided by Maryam Majeed), NeuroPAL (ot/s669)(YEMINI et al. 2021), ot/s356 (Is[rab-3::NLS::tagRFP])(SERRANO-SAIZ et al. 2013), Iqls4 [ceh-10p::GFP + lin-15(n765)] $X$ (kindly provided by Erik Lundquist), UL1692: unc-119(ed3); leEx1692 (Ex [h/h-34::gfp, unc-119(+)]) (CUNNINGHAM et al. 2012).

Fosmid-based reporters for $h / h-3, h l h-14$, lin-32 and cnd-1 were generated by insertion of gfp at the 3'end of their respective loci using fosmid recombineering (SAROV et al. 2012). Fosmid names are indicated in the figures. All fosmids were injected as complex arrays at the concentration of $20 \mathrm{ng} / \mu \mathrm{l}$ with $5 \mathrm{ng} / \mu \mathrm{l}$ of $t t x-3:: m$ Cherry as a co-injection marker and up to $85 \mathrm{ng} / \mu \mathrm{l}$ of OP50 DNA into N2 and then chromosomally integrated.

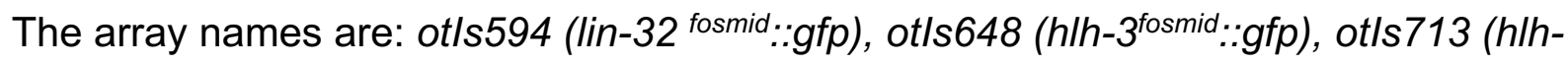

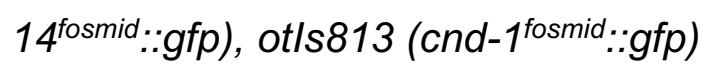

\section{Microscopy}

For fluorescence microscopy, worms were paralyzed by $25 \mathrm{mM}$ sodium azide $\left(\mathrm{NaN}_{3}\right)$ and mounted on $5 \%$ agarose pad on glass slides. Images were acquired using an 
axioscope (Zeiss, AXIO Imager Z.2) or LSM 800 laser point scanning confocal microscope. Representative images are max-projection of Z-stacks. Image reconstruction was performed using Fiji software (SCHINDELIN et al. 2012).

4D microscopy and SIMI BioCell (SCHNABEL et al. 1997) was used, as previously described, to analyze embryonic lineage defects of mutant animals as well as bHLH fosmid reporter expression pattern during embryogenesis. Briefly, gravid adults were dissected on glass slide and a single two-cell stage embryo was mounted and recorded over 8 hours of embryonic development. Nomarski stacks were taken every $30 \mathrm{~s}$ and embryos were illuminated with LED fluorescence light $(470 \mathrm{~nm})$ at set time points during development. The recording was done with Zeiss Imager Z1 compound microscope, using the 4D microscopy software Steuerprg (Caenotec). 


\section{RESULTS}

\section{Embryonic expression pattern of lin-32/Ato}

Even tough lin-32/Ato was identified more than a quarter century ago (ZHAO AND EMMONS 1995), its embryonic expression pattern has not been reported so far. We analyzed a transgenic strain carrying a fosmid in which the lin-32/Ato locus has been 3'terminally tagged with gfp, as well as as CRISPR/Cas9-engineered reporter allele, in which gfp was also inserted at the 3' end of the gene (kindly provided by the Greenwald lab), both of which yielded similar expression patterns. Embryonic expression was analyzed using 4D microscopy (SCHNABEL et al. 1997). We focused on the two lineages that produce all but two (PVR, DVC) of the 302 neurons of the nervous system, the $A B$ and MS blastomeres. We Fig.9 that lin-32/Ato expresses in a number of different neuronal lineages in the $A B$ lineage, but not the MS lineage, which produces only pharyngeal neurons (Fig.1). The earliest expression was observed at the 128-cell stage, in the daughters of the ABalapa blast cells. In other lineages the onset of lin32/Ato expression occurred shortly thereafter while, elsewhere, it commenced as late as a mother neuroblast that generates two terminally-differentiating daughters. In several cases we were not able to record the terminal division of a neuroblast due to the movement of embryos in the egg shell and we may therefore have missed expression in postmitotic cells. If postmitotic expression were to exist, it would be transient since we observed no expression of lin-32/Ato in any embryonically-generated neuron in first larval or later stage animals.

Consistent with expression of Atonal homologs in other organisms (BERTRAND et al. 2002; JARMAN AND GROVES 2013), we note that the vast majority of lineages that express lin-32/Ato produce sensory neurons. Several aspects of the embryonic expression of lin-32/Ato are in agreement with previously reported lin-32/Ato mutant phenotypes. For example, we observe lin-32/Ato expression (Fig.1) in lineages that give rise to sensory neurons in the anterior ganglion, consistent with previous studies that reported lin-32/Ato to control expression of the unc-86 terminal selector (BAUMEISTER et al. 1996; SHAHAM AND BARGMANN 2002). Similarly, we observed expression in lineages that give rise to the URX and CEPD neurons, and in lineages that give rise to the AIB 
neurons, which are all neurons wherein differentiation defects have been observed in lin-32/Ato mutants (DoITSIDOU et al. 2008; Rojo RomANOs et al. 2017; HoR et al. 2018).

\section{Proneural functions of lin-32/Ato}

Previous work had shown that lin-32/Ato has proneural functions in a number of postembryonically generated sensory neuronal cell types, including the sex-shared postdeirid lineage and the Q-lineage, as well as in male-specific neuronal lineages (Chalfie And Au 1989; Zhao ANd Emmons 1995; Portman ANd Emmons 2000). One common feature in these lineages appears to be that the loss of lin-32/Ato results in obvious lineage patterning defects, in which normally dividing neuroblasts transform into hypodermal cells. The embryonic expression of lin-32/Ato that we described above prompted us to ask whether such neuroblast-to-hypodermal conversions are also observed in embryonically generated neurons of lin-32/Ato null mutant animals (tm1449 deletion allele; Fig.1). Nomarksi optics-based lineage tracing of embryonic cell lineages was done using 4D microscopy with SIMI Biocell (SCHNABEL et al. 1997), until 300 minutes of embryonic development. This analysis revealed no obvious cell-division defects or transformations into hypodermal fates (as would be evidenced by changes in nuclear morphology and migratory patterns) in any of the lineages that normally express lin-32/Ato.

For a more granular assessment of cell fate, we examined the expression of different sets of panneuronal cell-fate markers. First, we used a nuclear, localized reporter transgene for the panneuronal rab-3 gene (STEFANAKIS et al. 2015). We counted the number of nuclei and, with the exception of the ventral nerve cord, observed a reduction in overall panneuronal gene expression throughout embryonically generated head and tail ganglia (Fig.2A). To further confirm this observation, we used a hybrid reporter construct in which the cis-regulatory elements of several panneuronally expressed genes are fused with one another, the "UPN" driver (YEMINI et al. 2021). We observed the expression of this panneuronal marker gene expression to also be lost in many different normally lin-32/Ato expressing lineages (Fig.2B,Fig.3). Lastly, we also examined the existence of subnuclear granules ("NUN bodies"), another panneuronal 
identity features (PHAM et al. 2021). We observed a reduction of the number of cells with this subnuclear structure (Fig.2C).

The hybrid panneuronal marker was expressed from the recently described NeuroPAL transgene, which also contains a large number of additional neuron-type specific markers (YEMINI et al. 2021). These markers allowed us to more specifically assess which neurons lose their identity and to correlate these losses with normal sites of lin-32/Ato expression. We observed panneuronal and cell-type specific marker losses in the AIB and URX neurons, which were previously reported to display differentiation defects in lin-32 mutants (RoJo RoMANOs et al. 2017; HoRI et al. 2018)(Fig.3). Within the anterior ganglion, we observed losses of panneuronal and cell-specific markers of the radially symmetric IL1 and IL2 sensory neuron classes, consistent with lin-32/Ato expression in the lineages that generate these neurons. We independently corroborated the IL1 and IL2 neuron losses with different marker transgenes ( $k / p-6$ for IL2 and flp-3 for IL1)(Suppl. Fig.S1).

In a previous screen for mutants that affect dopaminergic cell fate, we had identified alleles of lin-32/Ato and reported that lin-32/Ato affects the expression of the dopaminergic marker dat-1::gfp in the CEPD neurons (DoITSIDOU et al. 2008). Here, we found that lin-32/Ato is expressed in the lineage that gives rise to the CEPD neurons. Using NeuroPAL, we show that lin-32/Ato affects expression not only of the dat-1 marker, but of other cell-identity markers as well, in addition to panneuronal gene expression in CEPD. These results indicate that lin-32/Ato acts a proneural factor in this lineage as well (Fig.3).

\section{lin-32/Ato is required for terminal selector expression}

To further examine the nature of these differentiation defects, we asked whether lin-32/Ato controls the expression of the terminal selector-type transcription factors known to specify the identity of the neurons affected by lin-32/Ato. We found that the expression of unc-86, the identity regulator of the IL2 neurons and the URX neurons is indeed lost in lin-32/Ato mutants (Fig.4). Expression of ceh-43/D/x and ceh-32/Six, candidate terminal selectors for the IL1 neurons, and expression of $\operatorname{lin}-11 / \operatorname{Lh} x 1$, a 
candidate terminal selector for AVJ, are affected in the IL1 and AVJ neurons, respectively (Fig.4). Similarly, expression of ceh-43, the terminal selector for CEPD neurons (DoITSIDOU et al. 2013) is lost in CEPD of lin-32/Ato mutants (Fig.4), consistent with the loss of terminal CEPD identity markers in lin-32 mutants (Fig.3)(DoITSIDOU et al. 2008).

Since terminal selectors like unc-86, unc-42 and ceh-43 do not affect panneuronal gene expression (DOITSIDOU et al. 2013; HOBERT 2016; BERGHOFF et al. 2021), we conclude that lin-32/Ato independently regulates two aspects of the neuronal differentiation programs of cells like the IL2, IL1 or AIB neurons: panneuronal identity and the acquisition of neuron-type specific features via regulation of terminal-selector transcription factors.

\section{lin-32/Ato affects terminal identity markers in subsets of neuronal class members}

The effect of lin-32/Ato on a number of different neuron classes reveals an interesting phenomenon. All six IL2 class members are very similar neurons based on process projection patterns, synaptic connectivity (WHITE et al. 1986) and molecular markers (TAYLOR et al. 2020) and their common identity is specified by the terminal selectors unc-86, sox-2 and cfi-1 (SHAHAM AND BARGMANN 2002; ZHANG et al. 2014; VIDAL et al. 2015). However, we found that the lin-32/Ato mutant affects the differentiation of the dorsal and lateral IL2 pairs much more strongly than the ventral IL2 pairs (Fig.3, Fig.4). The subclass selective effect of lin-32/Ato correlates with the intriguing phenomenon that all six IL2 neurons derive from distinct lineages yet converge on the same neuron type. The dorsal and lateral IL2 pairs are all generated within the ABala lineage branch, while the ventral pairs are generated by the lineally distal ABalpp and ABarap branch (Fig.1). In the ABala branch, where loss of lin-32/Ato shows an effect, lin-32/Ato is normally expressed early in the lineage, while in the branches that produce the ventral pairs in a lin-32/Ato-independent manner, lin-32/Ato is expressed much later (Fig.1).

Another case of subclass expression is observed in the dopaminergic CEP neuron class, composed of a dorsal pair (CEPDL/R) and a ventral pair (CEPDVL/R). 
These two pairs are anatomically very similar (WHITE et al. 1986) and, in the terminally differentiated state, molecularly indistinguishable (TAYLOR et al. 2020). However, each pair derives from distinct embryonic neuroblasts (Fig.1). As described above, we found that lin-32/Ato has a proneural function in CEPD (Fig.3). However, even though lin32/Ato is expressed in the CEPV neurons (albeit much later than in the CEPD neuronproducing lineage; Fig.1), we detected no defect in the generation or differentiation of the CEPV neurons in lin-32/Ato null mutants. Panneuronal marker expression and terminal identity markers are unaffected (Fig.3) and expression of the terminal selector ceh-43 is also unaffected (Fig.4).

We observed another even more striking case of lin-32/Ato selectively affecting on lineally convergent neuron class members, relating to left/right symmetric neuron class members. Within the ABala lineage branch, the left and right AVH neurons are generated from two non-symmetric precursor cells, ABalapaaa (giving rise to AVHL) and ABalappap (giving rise to AVHR)(Fig.5A). Another neuron pair generated by these two different blast cells are CANL and CANR (Fig.5A). Like the six IL2 neurons, these two left/right symmetric neurons pairs within the ABala lineage are again examples of lineage convergence, where non bilaterally symmetric lineage histories funnel into the generation of indistinguishable left/right neuron pairs. We observed that lin-32/Ato is expressed in a left/right asymmetric manner in the lineages that gives rise to AVH and CAN neurons. In the lineage branch that gives rise to the left AVH and the left CAN, lin$32 /$ Ato is expressed throughout the lineage, while lin-32/Ato is expressed only very late in the postmitotic AVHR and not at all in CANR or the lineage that give rise to it (Fig.5). As stated above, we observed that lin-32/Ato does not affect the cellular cleavage pattern that gives rise to these cell types. However, we discovered that loss of lin-32/Ato affects, in a left/right asymmetric manner, the expression of terminal selector type transcription factors that define the molecular identity of neuron pairs within the ABala lineage. Specifically, lin-32/Ato affects expression of $h / h-34$, an identity regulator of the AVH neuron (BERGHOFF et al. 2021), in AVHL, where lin-32/Ato expression starts as early as the great-grandmother of AVHL, but not in AVHR, where we observe no lin32/Ato expression (Fig.5B). 
The same left/right symmetric effect is observed on the CAN neuron pair. CAN identity is specified by the Prd-type homeobox gene (FORRESTER et al. 1998; WENICK AND HOBERT 2004) and may operate together with the Distalless ortholog ceh-43 (REILLY et al. 2020). We find that lin-32/Ato affects expression of the CAN identity regulator ceh10 as well as its candidate cofactor ceh-43/Dlx in CANL, but not CANR (Fig.5B).

The achaete-scute homolog $h / h-14$ provides a mirror image of lin-32/Ato function

We considered the possibility that another bHLH transcription factor may provide a mirror-image function of lin-32/Ato in controlling the identity of neuron that are contralateral to those affected by lin-32/Ato. Unlike Drosophila or vertebrates, $C$. elegans only codes for a single Atonal ortholog (ZHAO AND EMMONS 1995; BAKER AND BROWN 2018). The next closely related bHLH genes are $n g n-1$ and $c n d-1$, the single Neurogenin and NeuroD orthologs of $C$. elegans which, together with Atonal, form the Ato superfamily of bHLH genes (HALLAM et al. 2000; HASSAN AND BELLEN 2000; NAKANO et al. 2010). We generated a strain with a fosmid reporter transgene for $c n d-1$. We also used a previously described $n g n-1$ reporter transgene that contains the entire $n g n-1$ locus and is capable of rescuing ngn-1 mutant phenotypes (NAKANO et al. 2010). These two strains were used to examine $c n d-1$ and $n g n-1$ expression throughout all stages of embryonic and postembryonic development of the hermaphrodite. We again focused on the $A B$ and MS lineages which produce all but 2 of the 118 neuron classes. We observed expression of both genes in several sublineages, with $n g n-1$ being more widespread than $c n d-1$ (Suppl. Fig.S2). ngn-1 and cnd-1 expression is largely nonoverlapping, with the exception of the ABarapp lineage where the expression of both genes overlaps (Suppl. Fig.S2). However, we observed no asymmetric expression within bilaterally symmetric neuron pairs in the ABala lineage that would mirror asymmetric expression of lin-32.

As the next gene candidates we considered the expression patterns of four homologs of the Drosophila Achaete-Scute complex (AS-C): hlh-6, hlh-19, hlh-3 and $h / h-14$. Their expression patterns had not previously been reported throughout the embryonic nervous system. A fosmid-based $h / h-6$ reporter is exclusively expressed in 
pharyngeal gland cells, as previously reported with smaller reporters (SMIT et al. 2008). We used CRISPR/Cas9 to tag endogenous $h / h-19$ with gfp and observed no expression at any stage of embryonic development. In contrast, an $h / h-3$ fosmid-based reporter, as well as a CRISPR-generated reporter allele (kindly provided by N. Flames), showed widespread expression throughout the developing embryonic nervous system (Fig.6). A hlh-14 fosmid reporter also showed embryonic expression in neuronal lineages, but its expression was more restricted (Fig.6). Intriguingly, in the context of the ABalap lineage where we observed left/right asymmetric lin-32/Ato expression (Fig.1), we observed that hlh-14 (but not hlh-3) displayed a mirror image asymmetry of this expression. In those left/right symmetric lineages where we observed differential later expression of lin32/Ato, we observed differential earlier expression of $h l h-14$, and vice versa (Fig.7A-C).

To assess the functional significance of this expression we examined two different left/right symmetric neuron pairs, CANL/R and AVHL/R in hlh-14 null mutants. We observed mirror image defects in hlh-14 mutants: CANR, but not CANL, loses ceh10 expression (the opposite phenotype as lin-32/Ato) and AVHR, but not AVHL, loses hlh-34 expression (also the opposite phenotype as lin-32/Ato)(Fig.7D). We further note that $h / h-14$ is also expressed as a mirror image in other lineages, particularly the IL1 and IL2 lineages, where lin-32/Ato shows differential expression in individual class members.

\section{Neuronal identity transformations in lin-32/Ato mutants}

Returning to our original analysis of lin-32/Ato mutants, we considered cases where we observed expression of lin-32/Ato, but no apparent loss of neuronal identity upon loss of lin-32. One example is the anterior deirid lineage which produces a group of five bilaterally symmetric neuron pairs (ADE, ADA, AIZ, FLP, RMG) and expresses lin-32/Ato early and uniformly (Fig.8A). We detected no proneural functions of lin-32/Ato in this lineage (i.e. no loss of the panneuronal marker) and we also observed no obvious defects of the cellular cleavage pattern in the lineage. A marker (dat-1::gfp) expressed in of one neuron class in the lineage, the dopaminergic ADE neuron, is expressed in several additional cells in lin-32/Ato mutants (DOITSIDOU et al. 2008), but it had been 
unclear whether these cells are ectopically generated cells or whether the marker is aberrantly expressed in other cells of this lineage. The use of NeuroPAL (YEMINI et al. 2021), which provides unique labels to all cells in the lineage, provided us with the opportunity to assess this defect in more detail. We independently confirmed the ectopic dopamine marker gene expression and found that it is paralleled by a loss of expression of markers for the AIZ, ADA, FLP and RMG neurons (Fig.8B,D). Hence, AIZ, ADA, FLP and RMG appear to have transformed their identity to that of the ADE neurons.

All of the 4 neuron classes that transform to ADE identity in lin-32/Ato mutants, normally express the unc-86/Brn3 POU homeobox gene (FINNEY AND RUVKUN 1990; SERRANO-SAIZ et al. 2018)(Fig.8A). unc-86/Brn3 acts as terminal selector in at least one of these neurons, FLP (TOPALIDOU AND CHALFIE 2011), and perhaps others as well (SERRANO-SAIz et al. 2013). We therefore tested whether loss of lin-32/Ato function affects unc-86 expression in these cells. Using a mNeonGreen-tagged unc-86 locus as a reporter for unc-86 expression (SERRANO-SAIZ et al. 2018), we indeed observed a loss of unc-86 expression in all 4 neuron classes of the lineage (Fig.8E). A similar result was also described previously using an antimorphic allele of lin-32/Ato, u282 (BAUMEISTER et al. 1996). Future work will determine whether the neuronal identity transformation observed in lin-32/Ato mutants in the anterior lineage is entirely explicable through the loss of unc-86 expression.

We observed another cell identity transformation in lin-32/Ato mutant animals in lineages that generate the four radially symmetric OLQ and URY neurons (Fig.9A). These neurons derive from four neuroblasts: ABalapapap, ABalapppap, and the two bilaterally symmetric $\mathrm{ABp}(\mathrm{l} / \mathrm{r})$ paaappp neurons. All of these neuroblasts were lin-32/Ato positive and maintained lin-32/Ato expression throughout ensuing divisions. As in the anterior deirid lineage, we found no proneural functions of lin-32/Ato in these lineages, i.e. we observed no obvious defects in cellular cleavage pattern in the lineage until the 300-minute stage (Supp Dataset 1), nor did we observe loss of panneuronal reporter expression. Instead, we observed another apparent neuronal identity transformation: OLQ neurons lose characteristic marker gene expression and instead gain expression of URY marker genes (Fig.9B-E). As in the anterior lineage, we sought to extend this observation by analyzing the expression of potential cell-identity regulators in this 
lineage. A potential URY identity regulator is the homeobox gene ceh-32/Six3, which is expressed in URY but not OLQ (REILLY et al. 2020). We observed that in lin-32/Ato mutants, OLQ neurons gain ceh-32 expression (Fig.9F). Taken together, we conclude that lin-32/Ato, either directly or indirectly, regulates the expression of terminal selectortype transcription factors that play a role in promoting specific neuronal identities and suppressing alternative identities. 


\section{DISCUSSION}

We found that, as expected from its previously described function in postembryonic development (ZHAO AND EMMONS 1995), lin-32/Ato acts as a proneural gene in a number of embryonically generated lineages. One distinguishing feature of the proneural role of lin-32/Ato in postembryonic versus embryonic development is that, in postembryonic development, a loss of the proneural activity of lin-32/Ato results in a termination of neuroblast divisions and conversion of the neuroblast to that of an ectodermal skin cells (ZHAO AND EMMONS 1995). In contrast, during embryogenesis, we found no evidence that the loss of lin-32/Ato affects cellular cleavage patterns or results in obvious conversions to hypodermal fate. What we do observe is a loss of expression of panneuronal features, a loss of cell identity-controlling transcription factors and, consequently, lost expression of cell-specific identity features.

One intriguing aspect of the proneural function of lin-32/Ato relates to the highly selective effect that lin-32/Ato has on specific members of individual neuron classes. This observation provides a molecular correlate to the phenomenon of lineage convergence: cells with different lineage histories acquire similar terminal identities. Lineage convergence is widespread in the C. elegans nervous system (SULSTON et al. 1983) and, with the advent of novel means to lineage more complex organisms, has now been observed in vertebrates as well (MCKENNA et al. 2016; WAGNER et al. 2018; CAO et al. 2019; CHAN et al. 2019). In the C. elegans nervous system, divergent lineages of individual neuron class members converge on similar gene expression profiles via the activation of terminal selectors of neuronal identity in individual class members. For example, the six IL2 sensory neurons arise from different, nonhomologous lineages but are all specified by the combinatorial activity of three terminal selectors, unc-86, cfi-1 and sox-2 (SHAHAM AND BARGMANN 2002; ZHANG et al. 2014; VIDAL et al. 2015). Likewise, the lineally diverse CEP neuron class members are all specified by the combinatorial activity of the terminal selectors ast-1, ceh-43 and ceh-20 (FLAMES AND HOBERT 2009; DoITSIDOU et al. 2013). We have shown here that different class members are specified by distinct upstream inputs. Specifically, lin-32/Ato controls the generation of distinct subsets of members from a given neuron class. In the most extreme cases, we observed that the left neuron of indistinguishable bilaterally 
symmetric neurons classes is controlled by lin-32/Ato. Intriguingly, the right neuron of these neuron classes is controlled by a distinct bHLH gene, $h / h-14$. Although we have not taken our study here to the cis-regulatory level, it is conceivable that the cisregulatory control regions of terminal selectors serve as "integration devices" that sample distinct lineage inputs.

Apart from proneural functions in a number of different cellular context, we also identified roles of lin-32/Ato in distinguishing the execution of distinct neuronal differentiation programs, such that the loss of lin-32/Ato leads to a conversion of one neuronal fate in that of another neuron fate. We observed such homeotic identity transformations in multiple distinct lineal contexts and we propose that these are also the result of lost terminal selector expression. Previous work has shown that in multiple different cellular contexts, terminal selectors can act in a mutually antagonistic and competitive manner, such that removal of one selector may now enable a different selector to exert its function (ARLOTTA AND HOBERT 2015). For example, loss of the mec3 homeobox gene in the ALM neuron allows UNC-86 to pair up with a different transcription factor, PAG-3, to now promote BDU neuron fate in the ALM neuron (WAY AND ChALFIE 1988; GoRdON AND HOBERT 2015). In analogy to these cases, it is conceivable that the lost expression of some terminal selectors in lin-32/Ato allows other terminal selectors to promote alternative fates.

In conclusion, we have provided insights into how terminal differentiation programs in the nervous system, controlled by terminal selector transcription factors, are coupled to earlier developmental events, and, specifically, to the lineage history of a cell. Our findings demonstrate that terminal selectors are key integrators of lineage history and provide novel perspectives on how proneuronal genes function to pattern the nervous system. 


\section{ACKNOWLEDGMENTS}

We thank Chi Chen for assistance with microinjections to generate strains, Cyril Cros, Maryam Majeed, Emily Berghoff, Nuria Flames, Erik Lundquist, Sarah Finkelstein, and Hana Littleford for kindly providing strains, and Iva Greenwald, Richard Poole and members of the Hobert lab for comments on the manuscript. This work was funded by the Howard Hughes Medical Institute. 


\section{FIGURE LEGENDS}

\section{Fig.1: lin-32/Ato expression pattern}

A: Schematic of gene structure shows that the deletion allele tm1664 removes the bHLH domain from lin-32/Ato locus.

B: Representative images of lin-32/Ato fosmid gene expression (ot/s594) at embryonic stages when most terminal neurons are born.

C: lin-32/Ato fosmid expression (ot/s594) in the AB and MS lineages, which produce all but 2 of the 118 neuron classes of the hermaphrodite. The lin-32/Ato fosmid reporter is first detected shortly after 100 minutes into development in ABalap descendants. Our analysis reveal that lin-32::gfp is expressed in both mitotically active neuroblasts during embryogenesis and a subset of postmitotic neurons.

Fig.2: Proneural activities of lin-32/Ato throughout the animal.

A: Expression of the panneuronal gene rab-3 is affected in different ganglia of the lin$32 /$ Ato null allele. The total number of cells expressing the rab-3 marker ot/s356 are plotted.

B: The total number of cells expressing the UPN panneuronal marker (contained within the NeuroPAL transgene ot/s669). The UPN reporter consist of 4 panneuronal promoters from ric-19, rgef-1, unc-11 and ehs-1 loci (YEMINI et al. 2021). We only scored UPN in the anterior ganglion.

C: The total number of cells with speckled subnuclear morphology ("NUN bodies") was scored in the anterior ganglion, using Nomarksi optics.

The error bars are indicating standard error of mean. Asterisks are indicating significance of $p<0.0001$. The statistical significance shown were calculated using twoway ANOVA with Tukey test for correction of multiple comparison.

\section{Fig.3: Proneuronal activities of lin-32/Ato in specific lineages}

A: The terminal and panneuronal identities of many neurons that express lin-32 during development are affected in lin-32(tm1446), as revealed here using the cell-fate marker 
strain NeuroPAL (ot/s669). Boxes with different color codes indicate the ganglion for neurons exhibiting fate defects.

B: The NeuroPAL reporter-fluorophore combinations responsible for distinguishably coloring each neurons type.

C: Statistics for terminal and panneuronal fate defects shown in panel A. The quantification shown here is the percentage of animals in which the wildtype NeuroPAL color code for the indicated neuron classes is observed in the respective neuron class, as revealed with the NeuroPAL (ot/s669) transgene. No instances were observed in which the terminal markers were affected while the panneuronal identity remained intact. The same effects of lin-32/Ato on dat-1 expression in CEPD (loss of expression) and CEPV (no effect detected) were also previously reported (DoITSIDOU et al. 2008). Circles indicate bilateral homologs of the respective neuron class $(L>0$ : Expression only in left neuron; $0<R$ : expression only in right neuron; $L=R$ : expression in both neurons = wildtype).

Fig.4: Effect of lin-32/Ato on the expression of candidate terminal selectors

A,B: Loss of candidate terminal selector expression in lin-32(tm1446) null mutants. Reporters are lin-11(ot958[lin-11::gfp::FLAG], ceh-32(ot1040[ceh-32::gfp]), unc86(ot879[unc-86::nNeonGreen]), ot/s339 (ceh-43fosmid::gfp) and unc-42 (ot958[unc42::gfp]) A: representative image; B: quantification. Circles indicate bilateral homologs of the respective neuron class ( $L>0$ : Expression only in left neuron; $0<R$ : expression only in right neuron; $\mathrm{L}=\mathrm{R}$ : expression in both neurons = wildtype).

\section{Fig.5: Left/right asymmetric neuronal defects in lin-32 mutants.}

A: ABalapa descendants are the very first blast cells to show expression of lin-32. The ABalapa lineage gives birth to two neurons, AVHL and CANL, among many other neurons. Conversely, their right contralateral homologs derive from the ABalapp lineage, which does not express lin-32/Ato.

B: Representative images of lin-32/Ato gene expression at embryonic stages displayed next to their exact time point during embryonic development: the $7^{\text {th }}$ (mitotically active cells) and $10^{\text {th }}$ (postmitotic cells) cleavage. The lower panel shows the ball model for the 
same embryonic stages. Color-coded boxes represent different regions of the embryo. The name of terminal and blast cells are indicated in the color-coded boxes.

C: The effect of lin-32(tm1446) was quantified using terminal selector reporters for AVH and CAN - lin-32/Ato is asymmetrically expressed in their ancestors.

\section{Fig.6: Embryonic expression of the AS-C homologs $h / h-3$ and $h / h-14$}

$h / h-3$ and $h / h-14$ fosmid reporters (ot/s648 and ot/s713, respectively) and their expression patterns.

A: Schematic of gene structure and fosmid reporters used for expression pattern analysis.

B: Representative images shown here are from $h / h-3$ and $h / h-14$ fosmid reporters during different stages of embryonic development; showing the time when the expression starts in blast cells to the time when all postmitotic neurons are born. Yellow asterisks are marking the cytoplasmic autofluorescence.

C: Lineage diagram showing the expression pattern of hlh-3 and hlh-14 during embryogenesis. Our analysis corroborates and extends the previously published expression patterns of hlh-14 (FRANK et al. 2003; POOLE et al. 2011).

\section{Fig.7: Left/right asymmetric neuronal defects in $h / h-14$ mutants}

A: The lineage diagram shows the expression of lin-32 and $h / h-14$ fosmid reporters in the ABala lineage side by side. hlh-14 mirrors the expression of lin-32 in ABalapa descendants, in ABalapp lineage. The dashed lines indicate sublineages that divide in a symmetric manner to produce symmetric cell fates, as shown in panel B.

B: A more focused version of the diagram in panel $A$, showing that $h l h-14$ expresses in the ABalapp descendants which give birth to AVHR and CANR, a mirror image of lin-32 expression (Fig.5).

C: Representative image of $h / h-14$ gene expression at the $7^{\text {th }}$ cleavage where mitotically active blast cells ABalappa/p (the great grandmother of AVHR and CANR) start expressing $h / h-14$. The right panel shows the ball model for the same embryonic stages. D: The effect of $h / h-14(t m 295)$ were quantified for terminal selector reporters of AVH 
and CAN - as was done for lin-32 mutants (Fig.5C). The wildtype images are from Fig $5 \mathrm{C}$ and are shown here for comparison only.

Fig.8: lin-32/Ato mutants display neuronal identity fate transformation in anterior deirid lineage

A: The expression of lin-32/Ato is shown in lineages giving birth to the anterior deirid neurons. The expression of lin-32/Ato overlaps perfectly with the previously reported unc-86 expression (FINNEY AND RUVKUN 1990).

B: The neuronal identities of Anterior deirid neurons were observed using NeuroPAL ot/s669. In lin-32(tm1446) mutants there are ectopic neurons expressing ADE- specific terminal markers while the terminal markers for FLP, RMG and sometimes the ADA neurons are lost. Quantification is shown in panel D.

C: The table shows the combination of different reporters in NeuroPAL strain used to mark cells in this lineage.

D: This panel shows the quantification of different phenotypic categories observed in lin32(tm1446) as compared to wildtype using NeuroPAL reporters. Circles indicate bilateral homologs of the respective neuron class $(L>0$ : Expression only in left neuron; $0<R$ : expression only in right neuron; $L=R$ : expression in both neurons = wildtype; $2 \mathrm{R}$ or 2L: expression in an additional neuron on left or right).

E: unc-86 expression (reporter allele ot879) is lost in the FLP, ADE and RMG neurons in the lin-32 mutants. Quantification is shown in right panel.

Fig.9: OLQ to URY identity transformation in lin-32/Ato null mutants

A: The lineage diagrams show the expression of lin-32/Ato in lineages giving birth to URY and OLQ neurons.

B: The terminal identity of OLQ and URY neurons is marked by NeuroPAL (ot/s669). In lin-32(tm1446) mutants there are ectopic signals for the terminal markers of URY while the OLQ specific signals were lost. Specifically, we observed potential duplicates of URYDR and URYVR in these mutants. Quantification is shown in panel I.

C: The table shows the combination of different reporters mixed in NeuroPAL strain used to mark URY and OLQ neurons. 
D: Correlation between the gain in URY-terminal markers and the loss of OLQ-terminal markers. URY and OLQ are lineal cousins of each other and their gain-loss correlation is statistically significant as tested by Pearson correlation ( $r=-0.534)$. The negative correlation value reflects the anticorrelated relationship between the observation of both types of neuron, meaning that observing URY is correlated with not observing its lineal cousin OLQ.

E: Quantification of NeuroPAL color codes observed in lin-32(tm1446) compared to the wildtype, using the NeuroPAL reporter (ot/s669). Left panels shows OLQ color code in ventral and dorsal OLQ neurons; this color code is partially lost in lin-32 mutants. Right panels shows URY color codes, which are observed in additional cells in lin-32 mutants. Circles indicate bilateral homologs of the respective neuron class ( $L>0$ : Expression only in left neuron; $0<R$ : expression only in right neuron; $L=R$ : expression in both neurons = wildtype; 2R or 2L: expression in an additional neuron on left or right).

F: Ectopic OLQ expression of the ceh-32 reporter allele (ot1040), normally expressed in URY. Quantification is shown in right panel. 


\section{SUPPLEMENTARY LEGENDS}

Suppl. Dataset 1: Lineage movie of lin-32/Ato animals. Two embryos from lin32(tm1446) were lineaged using the classic Nomarski-based technique (SCHNABEL et al. 1997). The movies are in Laura Waivier format and can be seen using SIMIBiocell software.

\section{Suppl. Fig.S1: Additional effects of lin-32/Ato on marker gene expression.}

The terminal identity of IL1 and IL2 neurons are affected in lin-32(tm1446), shown here using flp-3::mCherry and klp-6::gfp reporters. Quantification is shown in lower panels. Circles indicate bilateral homologs of the respective neuron class $(L>0$ : Expression only in left neuron; $0<R$ : expression only in right neuron; $L=R$ : expression in both neurons = wildtype).

\section{Suppl. Fig.S2: Embryonic expression of the C. elegans neurogenin and NeuroD homologs, ngn-1 and cnd-1.}

cnd-1 and ngn-1 reporters (ot/s813 and $n / s 394$, respectively) and their expression patterns.

A: Schematic of gene structure and the fosmid used for expression pattern analysis.

B: Representative images of $c n d-1$ and $n g n-1$ gene expression at embryonic stages, starting from the earliest stage where the expression starts and continuing to the time when all terminal neurons have been born. Yellow asterisk is marking the cytoplasmic autofluorescence and red arrows are pointing toward real expression inside nuclei. C: The full lineage of $c n d-1$ and ngn-1 expression in the AB and MS lineages; these lineages produce all but 2 of the 302 neurons in adult $C$. elegans hermaphrodites. Our analysis confirms previously published expression patterns reported for a subset of the cells shown here (HALLAM et al. 2000; NAKANO et al. 2010). 


\section{References}

Arlotta, P., and O. Hobert, 2015 Homeotic Transformations of Neuronal Cell Identities. Trends Neurosci 38: 751-762.

Baker, N. E., and N. L. Brown, 2018 All in the family: proneural bHLH genes and neuronal diversity. Development 145.

Baumeister, R., Y. Liu and G. Ruvkun, 1996 Lineage-specific regulators couple cell lineage asymmetry to the transcription of the Caenorhabditis elegans POU gene unc-86 during neurogenesis. Genes Dev 10: 1395-1410.

Berghoff, E., L. Glenwinkel, A. Bhattacharya, H. Sun, N. Mohammadi et al., 2021 The Prop1-like homeobox gene unc-42 specifies the identity of

synaptically connected neurons. eLife in revision.

Bertrand, N., D. S. Castro and F. Guillemot, 2002 Proneural genes and the specification of neural cell types. Nat Rev Neurosci 3: 517-530.

Bertrand, V., and O. Hobert, 2009 Linking asymmetric cell division to the terminal differentiation program of postmitotic neurons in C. elegans. Dev Cell 16: 563575.

Brenner, S., 1974 The genetics of Caenorhabditis elegans. Genetics 77: 71-94.

Cao, J., M. Spielmann, X. Qiu, X. Huang, D. M. Ibrahim et al., 2019 The single-cell transcriptional landscape of mammalian organogenesis. Nature 566: 496-502.

Chalfie, M., and M. Au, 1989 Genetic control of differentiation of the Caenorhabditis elegans touch receptor neurons. Science 243: 1027-1033.

Chan, M. M., Z. D. Smith, S. Grosswendt, H. Kretzmer, T. M. Norman et al., 2019 Molecular recording of mammalian embryogenesis. Nature 570: 77-82.

Cunningham, K. A., Z. Hua, S. Srinivasan, J. Liu, B. H. Lee et al., 2012 AMP-activated kinase links serotonergic signaling to glutamate release for regulation of feeding behavior in C. elegans. Cell Metab 16: 113-121.

Doitsidou, M., N. Flames, A. C. Lee, A. Boyanov and O. Hobert, 2008 Automated screening for mutants affecting dopaminergic-neuron specification in $\mathrm{C}$. elegans. Nat Methods 5: 869-872.

Doitsidou, M., N. Flames, I. Topalidou, N. Abe, T. Felton et al., 2013 A combinatorial regulatory signature controls terminal differentiation of the dopaminergic nervous system in C. elegans. Genes Dev 27: 1391-1405.

Finney, M., and G. Ruvkun, 1990 The unc- 86 gene product couples cell lineage and cell identity in C. elegans. Cell 63: 895-905.

Flames, N., and O. Hobert, 2009 Gene regulatory logic of dopamine neuron differentiation. Nature 458: 885-889.

Forrester, W. C., E. Perens, J. A. Zallen and G. Garriga, 1998 Identification of Caenorhabditis elegans genes required for neuronal differentiation and migration. Genetics 148: 151-165.

Frank, C. A., P. D. Baum and G. Garriga, $2003 \mathrm{HLH}-14$ is a C. elegans achaete-scute protein that promotes neurogenesis through asymmetric cell division. Development 130: 6507-6518.

Gordon, P. M., and O. Hobert, 2015 A Competition Mechanism for a Homeotic Neuron Identity Transformation in C. elegans. Dev Cell 34: 206-219. 
Hallam, S., E. Singer, D. Waring and Y. Jin, 2000 The C. elegans NeuroD homolog cnd1 functions in multiple aspects of motor neuron fate specification. Development 127: 4239-4252.

Hassan, B. A., and H. J. Bellen, 2000 Doing the MATH: is the mouse a good model for fly development? Genes Dev 14: 1852-1865.

Hobert, O., 2016 Terminal Selectors of Neuronal Identity. Curr Top Dev Biol 116: 455475.

Hobert, O., L. Glenwinkel and J. White, 2016 Revisiting Neuronal Cell Type Classification in Caenorhabditis elegans. Curr Biol 26: R1197-R1203.

Hori, S., S. Oda, Y. Suehiro, Y. lino and S. Mitani, 2018 OFF-responses of interneurons optimize avoidance behaviors depending on stimulus strength via electrical synapses. PLoS Genet 14: e1007477.

Jarman, A. P., and A. K. Groves, 2013 The role of Atonal transcription factors in the development of mechanosensitive cells. Semin Cell Dev Biol 24: 438-447.

McKenna, A., G. M. Findlay, J. A. Gagnon, M. S. Horwitz, A. F. Schier et al., 2016 Whole-organism lineage tracing by combinatorial and cumulative genome editing. Science 353: aaf7907.

Miller, R. M., and D. S. Portman, 2011 The Wnt/beta-catenin asymmetry pathway patterns the atonal ortholog lin-32 to diversify cell fate in a Caenorhabditis elegans sensory lineage. J Neurosci 31: 13281-13291.

Mitani, S., H. Du, D. H. Hall, M. Driscoll and M. Chalfie, 1993 Combinatorial control of touch receptor neuron expression in Caenorhabditis elegans. Development 119: 773-783.

Murgan, S., W. Kari, U. Rothbacher, M. Iche-Torres, P. Melenec et al., 2015 Atypical Transcriptional Activation by TCF via a Zic Transcription Factor in C. elegans Neuronal Precursors. Dev Cell 33: 737-745.

Nakano, S., R. E. Ellis and H. R. Horvitz, 2010 Otx-dependent expression of proneural bHLH genes establishes a neuronal bilateral asymmetry in C. elegans. Development 137: 4017-4027.

Packer, J. S., Q. Zhu, C. Huynh, P. Sivaramakrishnan, E. Preston et al., 2019 A lineage-resolved molecular atlas of $\mathrm{C}$. elegans embryogenesis at single-cell resolution. Science 365.

Pham, K., N. Masoudi, E. Leyva-Diaz and O. Hobert, 2021 A nervous system-specific subnuclear organelle in Caenorhabditis elegans. Genetics in press.

Poole, R. J., E. Bashllari, L. Cochella, E. B. Flowers and O. Hobert, 2011 A GenomeWide RNAi Screen for Factors Involved in Neuronal Specification in Caenorhabditis elegans. PLoS Genet 7: e1002109.

Portman, D. S., and S. W. Emmons, 2000 The basic helix-loop-helix transcription factors LIN-32 and HLH-2 function together in multiple steps of a C. elegans neuronal sublineage. Development 127: 5415-5426.

Reilly, M. B., C. Cros, E. Varol, E. Yemini and O. Hobert, 2020 Unique homeobox codes delineate all the neuron classes of C. elegans. Nature 584: 595-601.

Rojo Romanos, T., D. Pladevall-Morera, K. Langebeck-Jensen, S. Hansen, L. Ng et al., 2017 LIN-32/Atonal Controls Oxygen Sensing Neuron Development in Caenorhabditis elegans. Sci Rep 7: 7294. 
Sarov, M., J. I. Murray, K. Schanze, A. Pozniakovski, W. Niu et al., 2012 A genomescale resource for in vivo tag-based protein function exploration in $\mathrm{C}$. elegans. Cell 150: 855-866.

Schindelin, J., I. Arganda-Carreras, E. Frise, V. Kaynig, M. Longair et al., 2012 Fiji: an open-source platform for biological-image analysis. Nat Methods 9: 676-682.

Schnabel, R., H. Hutter, D. Moerman and H. Schnabel, 1997 Assessing normal embryogenesis in Caenorhabditis elegans using a 4D microscope: variability of development and regional specification. Dev Biol 184: 234-265.

Serrano-Saiz, E., E. Leyva-Diaz, E. De La Cruz and O. Hobert, 2018 BRN3-type POU Homeobox Genes Maintain the Identity of Mature Postmitotic Neurons in Nematodes and Mice. Curr Biol 28: 2813-2823 e2812.

Serrano-Saiz, E., Richard J. Poole, T. Felton, F. Zhang, Estanisla D. De La Cruz et al., 2013 Modular Control of Glutamatergic Neuronal Identity in C. elegans by Distinct Homeodomain Proteins. Cell 155: 659-673.

Shaham, S., and C. I. Bargmann, 2002 Control of neuronal subtype identity by the C. elegans ARID protein CFI-1. Genes Dev 16: 972-983.

Smit, R. B., R. Schnabel and J. Gaudet, 2008 The HLH-6 transcription factor regulates C. elegans pharyngeal gland development and function. PLoS Genet 4: e1000222.

Stefanakis, N., I. Carrera and O. Hobert, 2015 Regulatory Logic of Pan-Neuronal Gene Expression in C. elegans. Neuron 87: 733-750.

Sulston, J. E., and H. R. Horvitz, 1977 Post-embryonic cell lineages of the nematode, Caenorhabditis elegans. Dev Biol 56: 110-156.

Sulston, J. E., E. Schierenberg, J. G. White and J. N. Thomson, 1983 The embryonic cell lineage of the nematode Caenorhabditis elegans. Dev Biol 100: 64-119.

Taylor, S. R., G. Santpere, A. Weinreb, A. Barrett, M. Reilly et al., 2020 Molecular topography of an entire nervous system. BioRxiv.

Topalidou, I., and M. Chalfie, 2011 Shared gene expression in distinct neurons expressing common selector genes. Proc Natl Acad Sci U S A 108: 1925819263.

Vidal, B., A. Santella, E. Serrano-Saiz, Z. Bao, C. F. Chuang et al., 2015 C. elegans SoxB genes are dispensable for embryonic neurogenesis but required for terminal differentiation of specific neuron types. Development 142: 2464-2477.

Wagner, D. E., C. Weinreb, Z. M. Collins, J. A. Briggs, S. G. Megason et al., 2018 Single-cell mapping of gene expression landscapes and lineage in the zebrafish embryo. Science 360: 981-987.

Way, J. C., and M. Chalfie, 1988 mec-3, a homeobox-containing gene that specifies differentiation of the touch receptor neurons in C. elegans. Cell 54: 5-16.

Wenick, A. S., and O. Hobert, 2004 Genomic cis-Regulatory Architecture and transActing Regulators of a Single Interneuron-Specific Gene Battery in C. elegans. Dev Cell 6: 757-770.

White, J. G., E. Southgate, J. N. Thomson and S. Brenner, 1986 The structure of the nervous system of the nematode Caenorhabditis elegans. Philosophical Transactions of the Royal Society of London B. Biological Sciences 314: 1-340. 
Yemini, E., A. Lin, A. Nejatbakhsh, E. Varol, R. Sun et al., 2021 NeuroPAL: A Multicolor Atlas for Whole-Brain Neuronal Identification in C. elegans. Cell 184: 272-288 e211.

Zhang, A., K. Noma and D. Yan, 2020 Regulation of Gliogenesis by lin-32/Atoh1 in Caenorhabditis elegans. G3 (Bethesda) 10: 3271-3278.

Zhang, F., A. Bhattacharya, J. C. Nelson, N. Abe, P. Gordon et al., 2014 The LIM and POU homeobox genes ttx-3 and unc-86 act as terminal selectors in distinct cholinergic and serotonergic neuron types. Development 141: 422-435.

Zhao, C., and S. W. Emmons, 1995 A transcription factor controlling development of peripheral sense organs in C. elegans. Nature 373: 74-78.

Zhu, Z., J. Liu, P. Yi, D. Tian, Y. Chai et al., 2014 A proneural gene controls C. elegans neuroblast asymmetric division and migration. FEBS Lett 588: 1136-1143. 

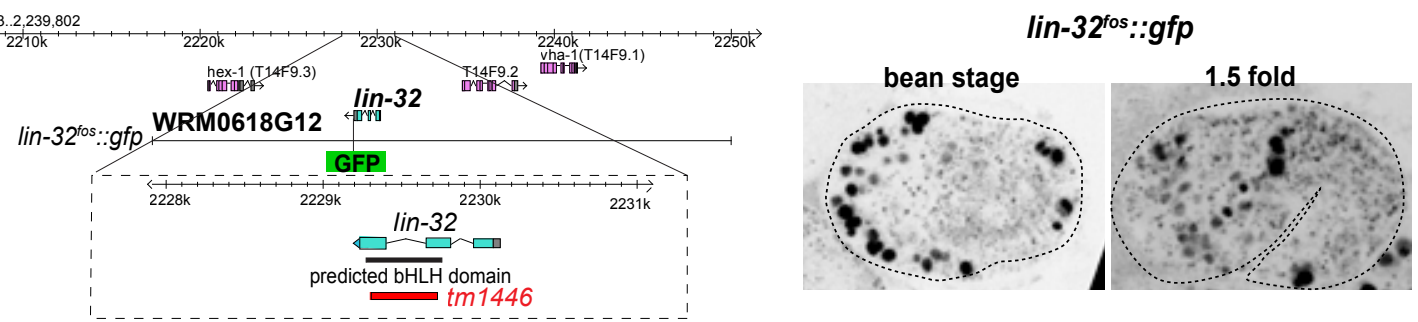

C

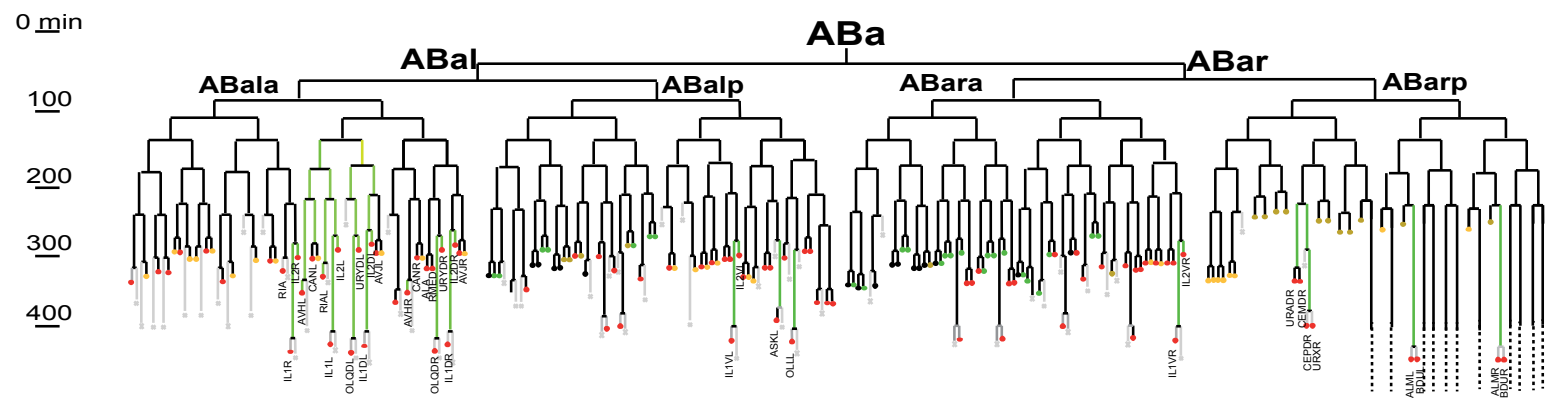

$0 \underline{\min }$

$1 \underline{00}$

ABp

$\underline{20}$
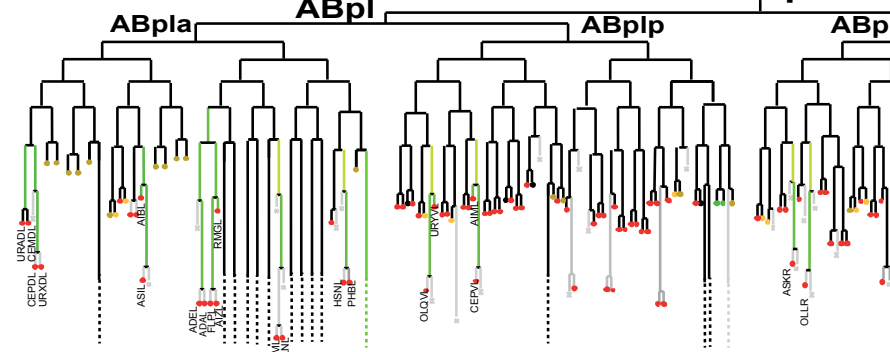

ABpra

ABpr

ABprp

30

$\underline{40}$

$0 \underline{\min }$

MS

$\underline{100}$

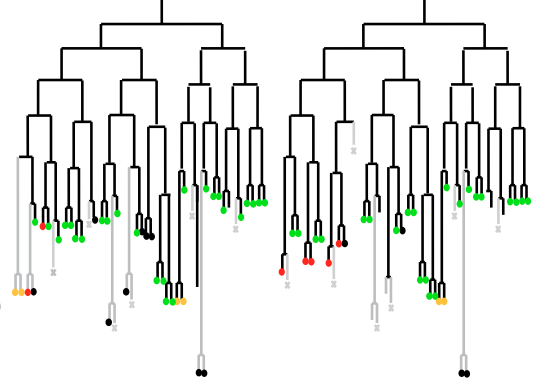

- lin-32 dim expression

lin-32 strong expression

200

$\underline{300}$

Figure 1 


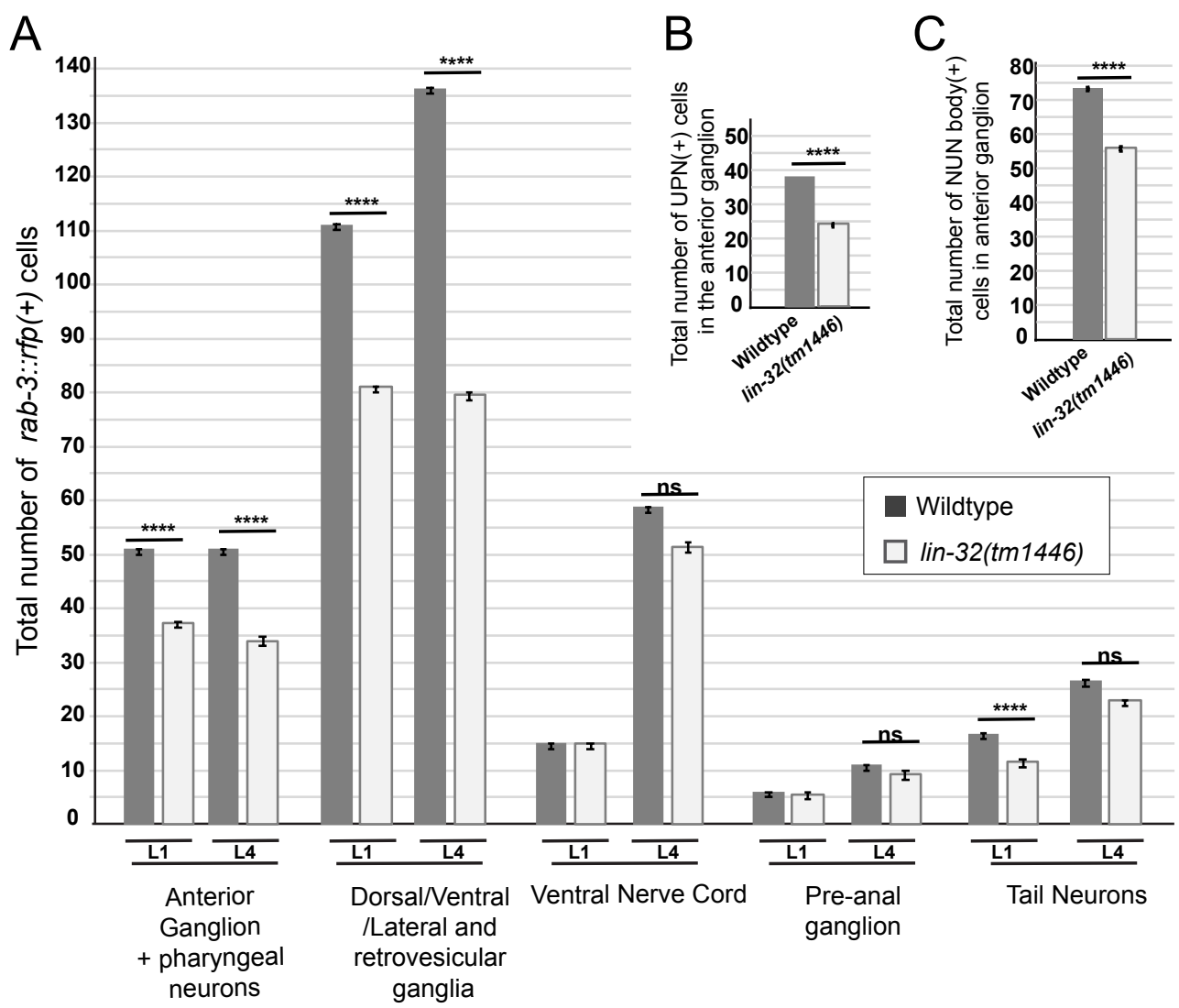

Figure 2 


\section{A}

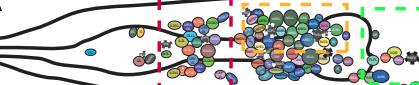

Terminal Panneuronal Terminal Panneurona

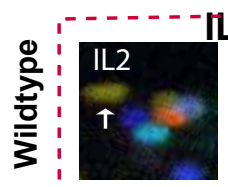

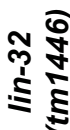

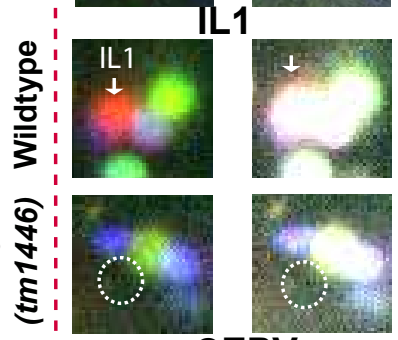

C

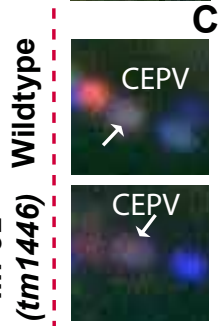

CEPV

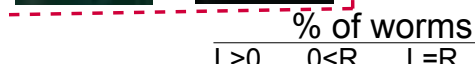

$\begin{array}{lllll}L>0 & 0<R & L=R & \text { no exp }\end{array}$

0 .

额 $\begin{array}{cccccc}\mathbf{N} 2 & 0 & 0 & 100 & 0 & 30 \\ \text { lin-32 } & 20 & 36.7 & 33.3 & 10 & 30\end{array}$

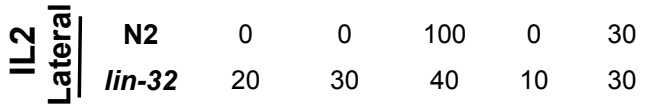

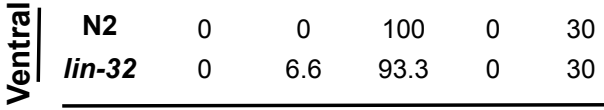

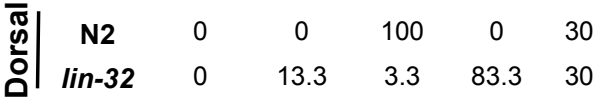

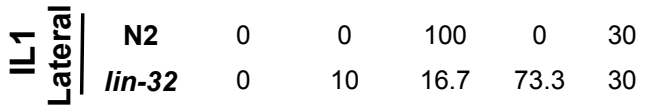

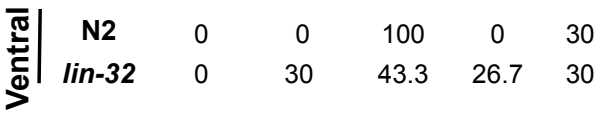

Terminal Panneuronal Terminal Panneuronal
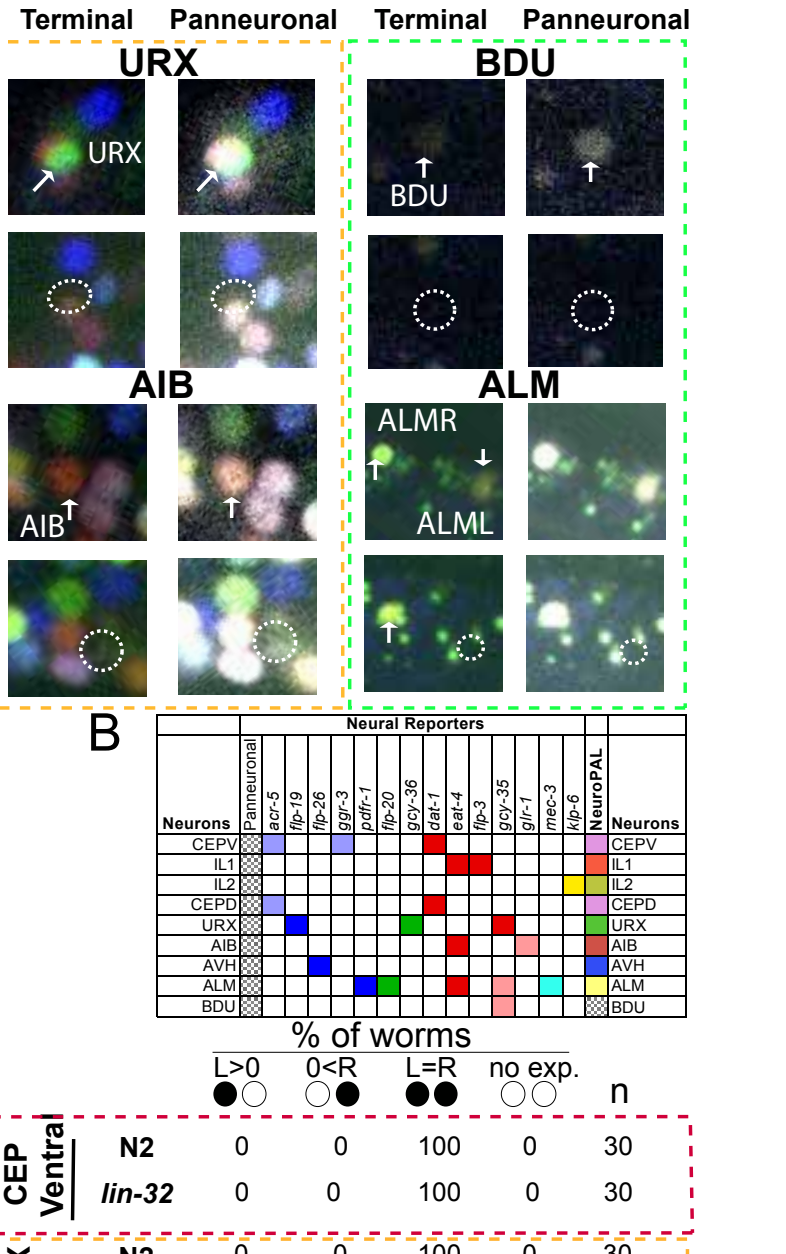

ALM

BDU
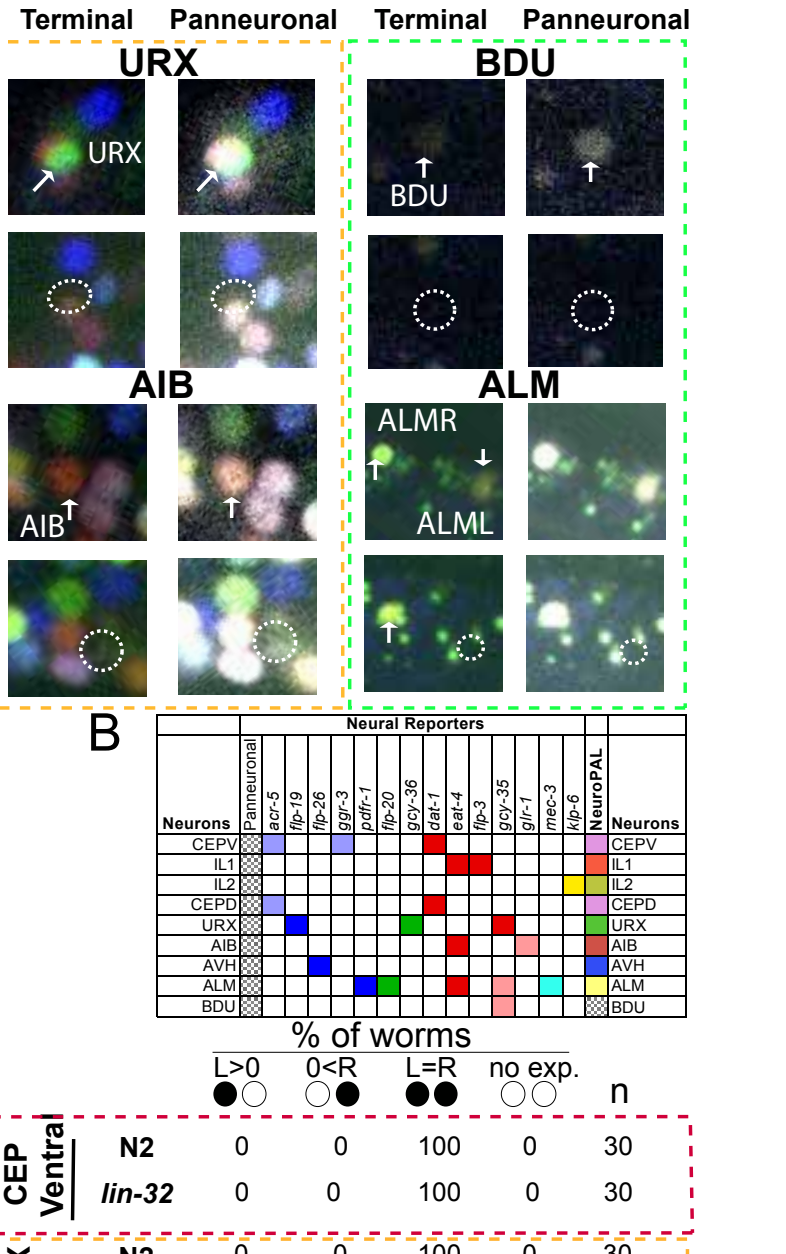

B

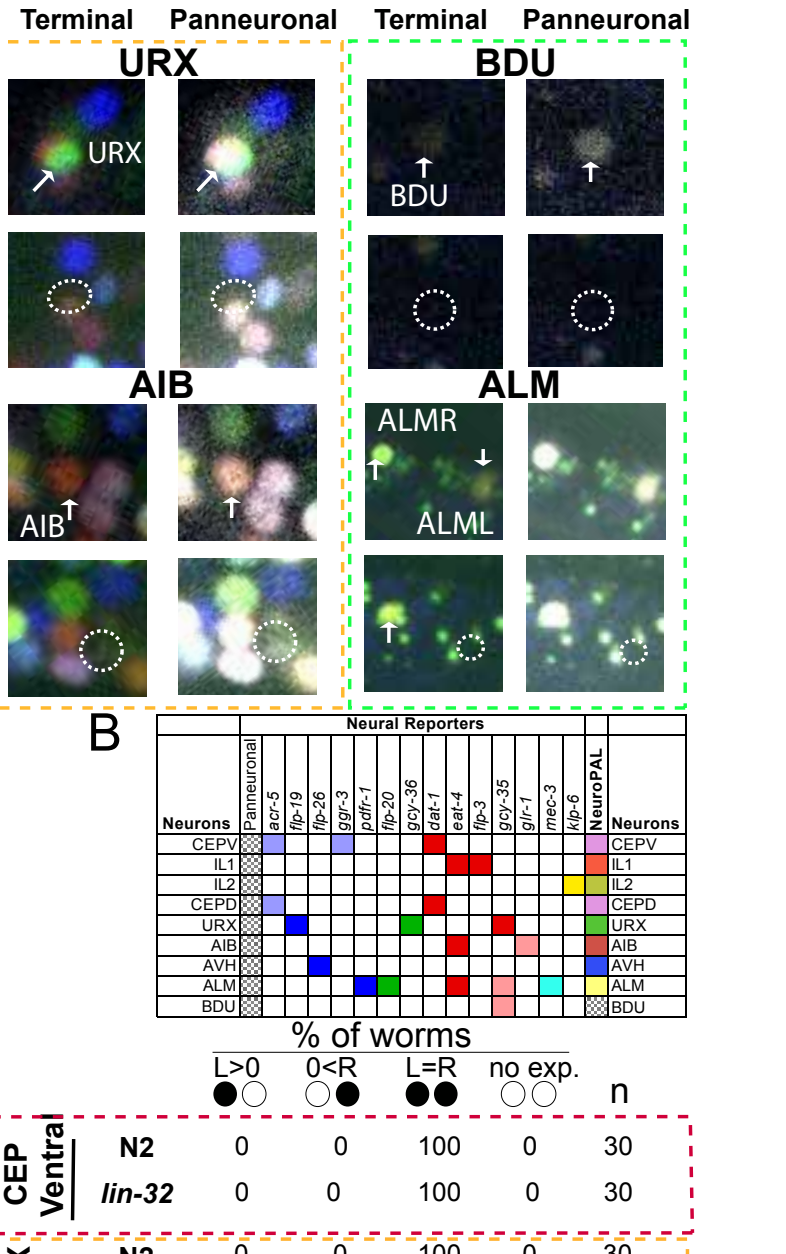

$\mathrm{L}>0 \quad 0<\mathrm{R} \quad \mathrm{L}=\mathrm{R} \quad$ no exp.

0 O० 00 n

:

X

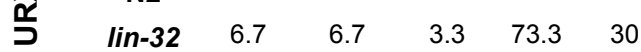

$\begin{array}{lllllll}\overline{\text { ֻ̆ }} & \text { N2 } & 0 & 0 & 100 & 0 & 30\end{array}$

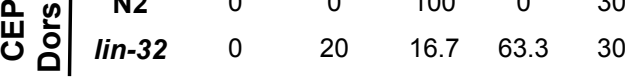

\begin{tabular}{ccccccc}
\cline { 2 - 6 } & $\mathbf{N} 2$ & 0 & 0 & 100 & 0 & 30 \\
& lin-32 & 0 & 56.7 & 40 & 3.33 & 30
\end{tabular}

$\begin{array}{lllllll}\boldsymbol{m} & \mathbf{N 2} & 0 & 0 & 100 & 0 & 30\end{array}$

\begin{tabular}{lllllll}
\hline & lin-32 & 16.7 & 20 & 50 & 13.3 & 30
\end{tabular}

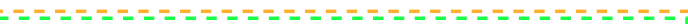

$\begin{array}{lllllll}7 & \text { N2 } & 0 & 0 & 100 & 0 & 30\end{array}$

$\begin{array}{lllllll}\boldsymbol{m} & \text { lin-32 } & 13.3 & 26.7 & 36.7 & 20 & 30\end{array}$

\begin{tabular}{ccccccc:c}
\hline $\mathbf{N} 2$ & 0 & 0 & 100 & 0 & 30 & \\
lin-32 & 16.7 & 13.3 & 63.3 & 6.7 & 30 & Fig.3
\end{tabular}




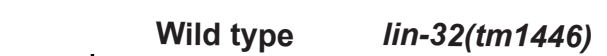

Wild type lin-32(tm1446)
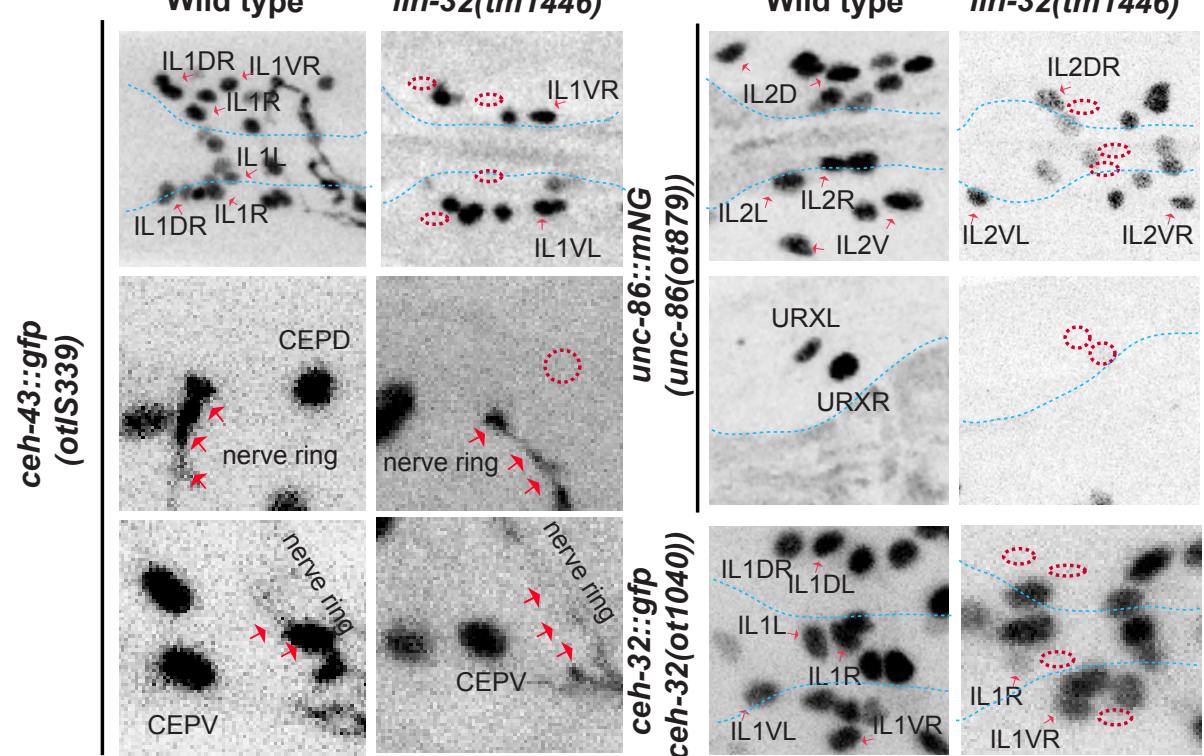

B
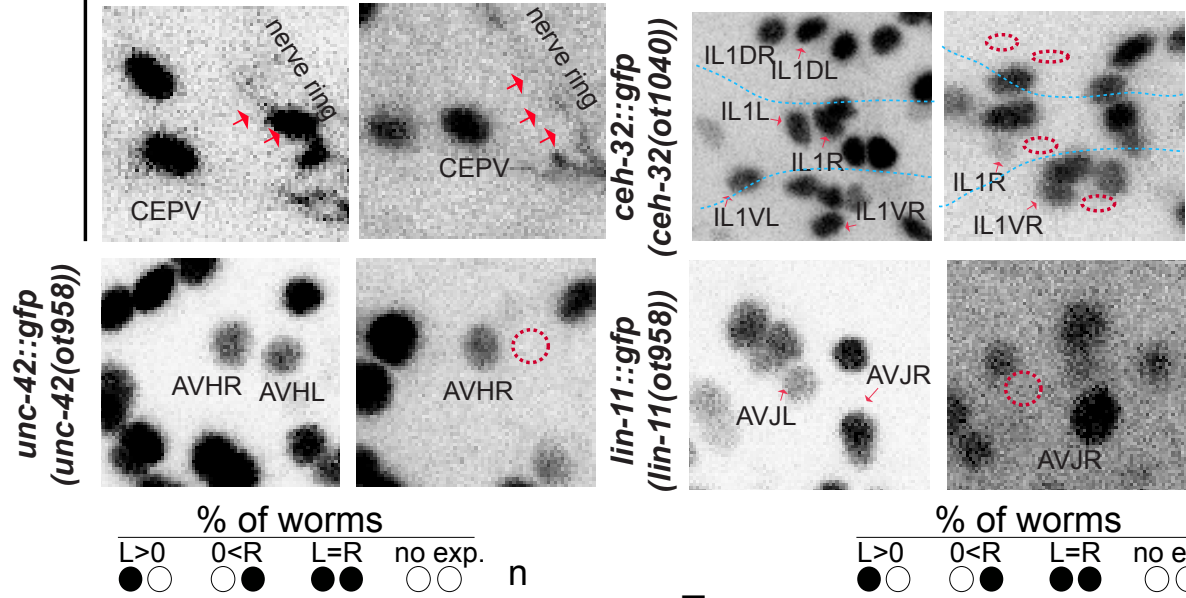

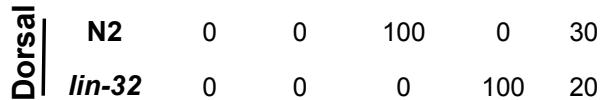

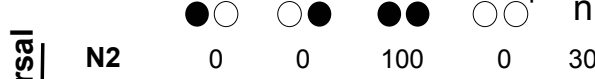

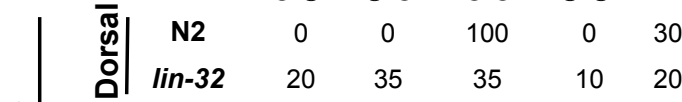

$\begin{array}{lllllll}-\overline{\bar{T}} & \text { N2 } & 0 & 0 & 100 & 0 & 30\end{array}$

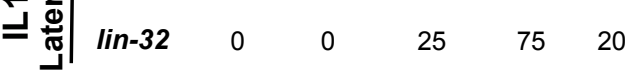

ॠ $\begin{array}{llllll}\text { N2 } & 0 & 0 & 100 & 0 & 30\end{array}$ $\begin{array}{llllll}\text { 今े lin-32 } & 0 & 15 & 10 & 75 & 20\end{array}$

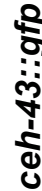

$\begin{array}{lllllll}\text { 胥 } & \text { N2 } & 0 & 0 & 100 & 0 & 30\end{array}$

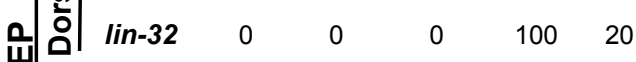

క.

$\begin{array}{lllllll}\mathbf{T} \frac{\bar{T}}{0} & N 2 & 0 & 0 & 100 & 0 & 30\end{array}$

0

$\begin{array}{lllllll}\overline{\sqrt{2}} & \mathbf{N} 2 & 0 & 0 & 100 & 0 & 30\end{array}$

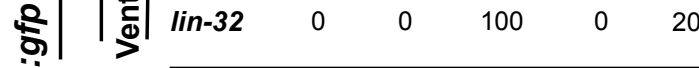

گ̇

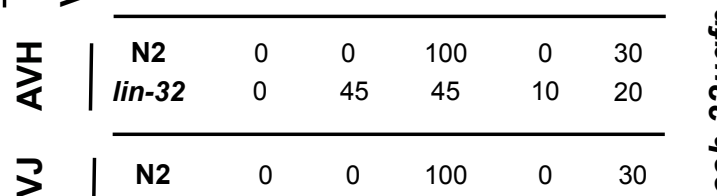

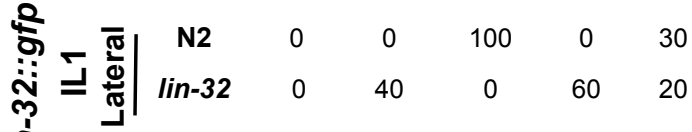

䒿 $\begin{array}{llllll}\text { N2 } & 0 & 0 & 100 & 0 & 30\end{array}$

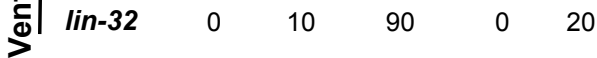

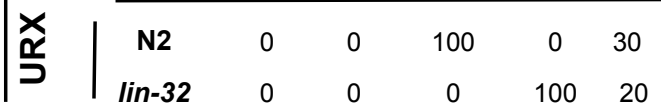

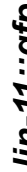

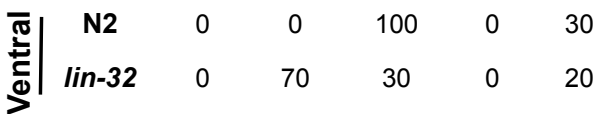



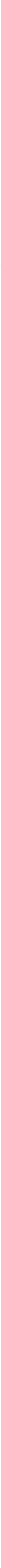

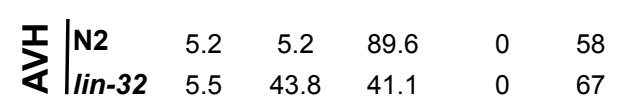

Fig.5 
II

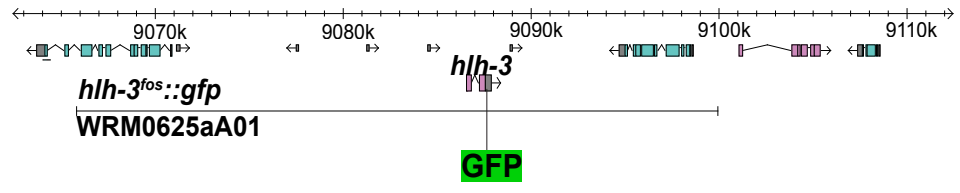

II

\begin{tabular}{|c|c|c|c|c|c|}
\hline 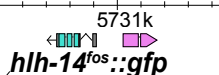 & 5736k & $\begin{array}{l}h l h-14 \\
4 \square>\square\end{array}$ & $5741 \mathrm{k}$ & साप & $5746 \mathrm{k}$ \\
\hline $\begin{array}{l}\text { WRM0627dH07 } \\
\text { (Sarov fosmid) }\end{array}$ & & GFI & & & \\
\hline
\end{tabular}
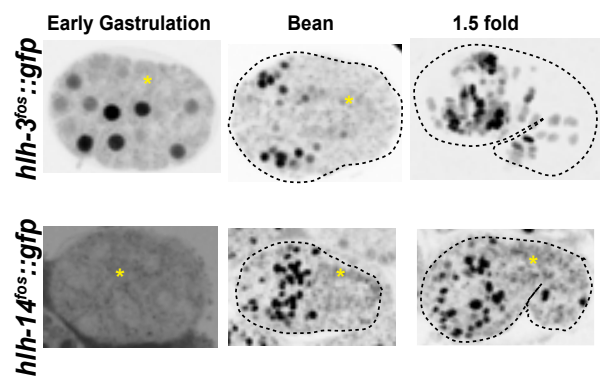

C

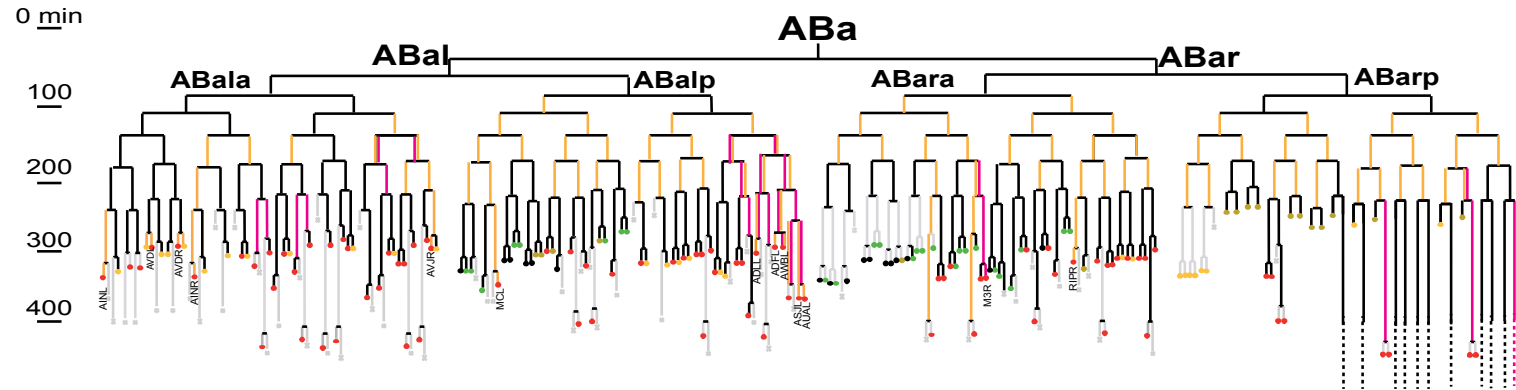

0 min
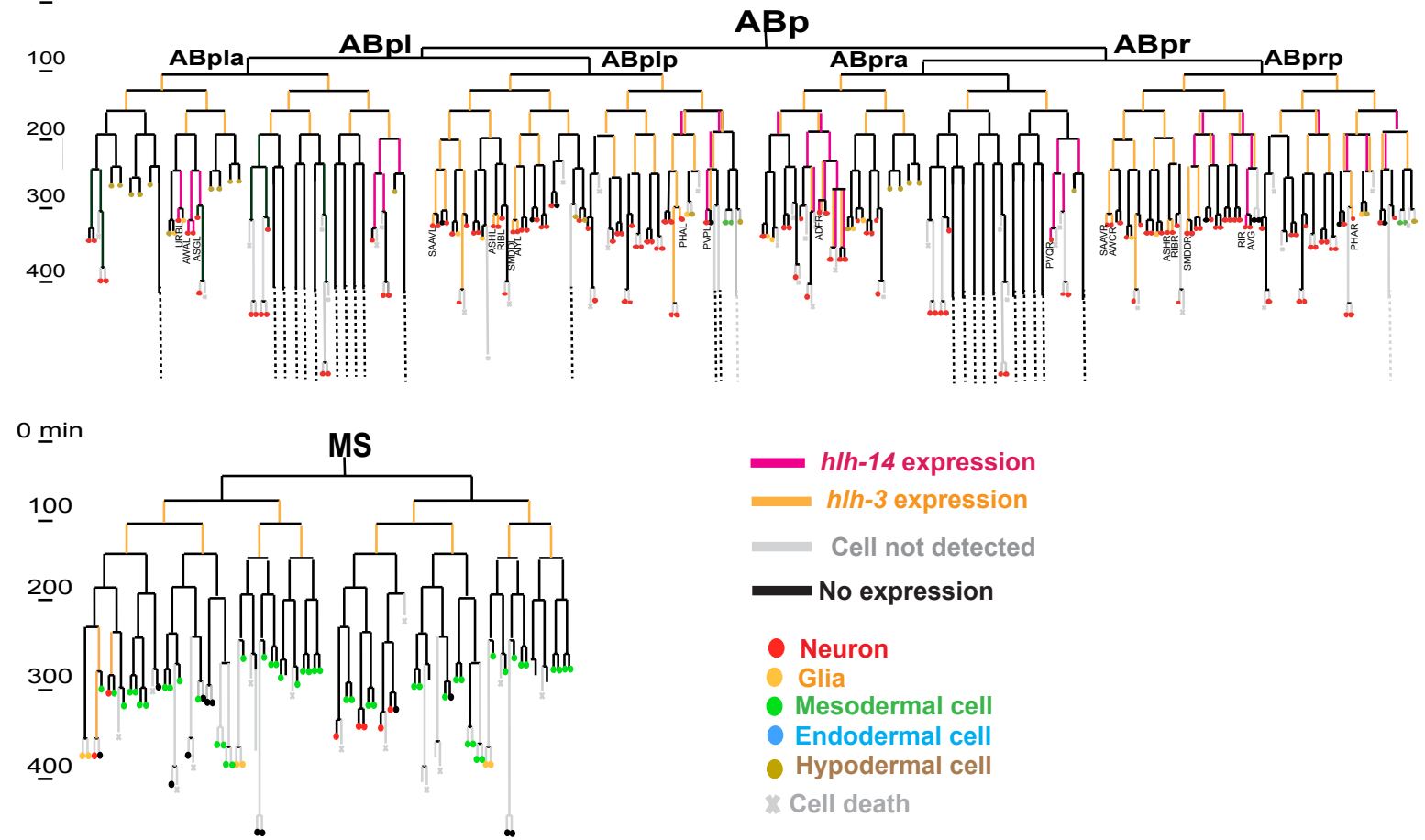

Wh-14 expression

h/h-3 expression

Cell not detected

No expression

Neuron

Glia

- Mesodermal cell

- Endodermal cell

- Hypodermal cell

* Cell death 


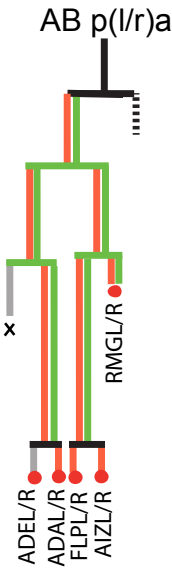

| lin-32::gfp

unc-86 antibody staining

$\%$ of worms expressing NeuroPAL eporters $\stackrel{L}{\longrightarrow} \quad 0<R \quad L=R \quad$ no exp. $n$
\begin{tabular}{c|ccccc}
$\mathbf{2} 2$ & 0 & 0 & 100 & 0 & 30 \\
lin-32 & 23.3 & 30 & 40 & 6.7 & 30
\end{tabular}

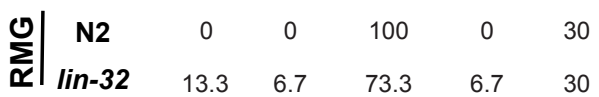

E

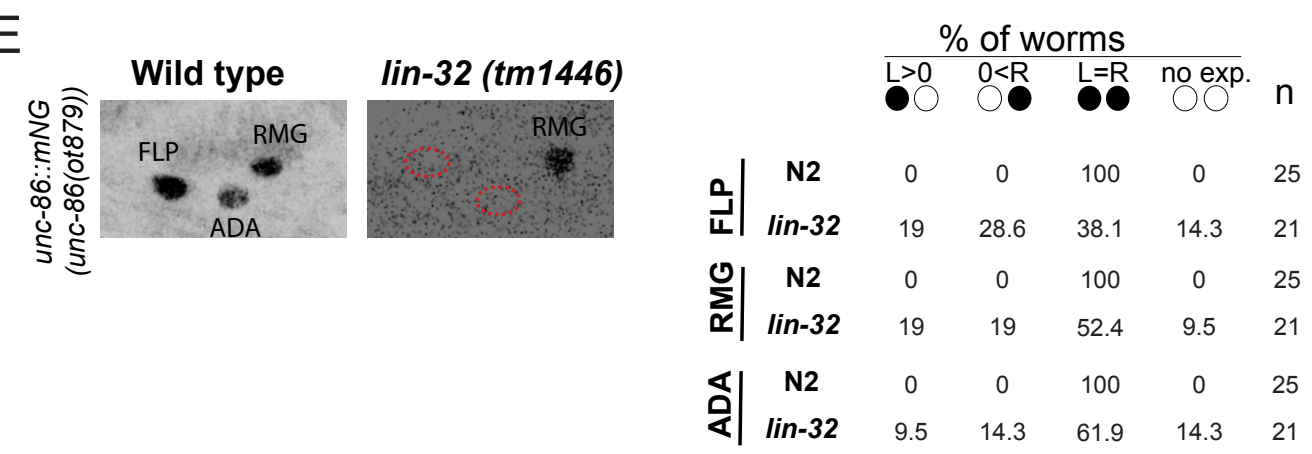

Wild type

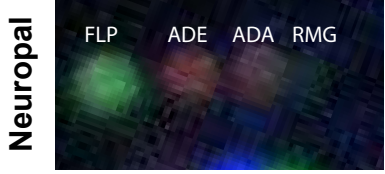

lin-32(tm1446)

\begin{tabular}{|c|c|c|c|c|c|c|}
\hline & & iral & $\operatorname{Repc}$ & orter & & \\
\hline Neurons & 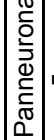 & $\begin{array}{l}\frac{1}{1} \\
\substack{0 \\
0}\end{array}$ & 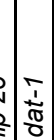 & $\mid$ & & Neurons \\
\hline ADA & & & & & & ADA \\
\hline ADE & & & & & & ADE \\
\hline FLP & & & & & & FLP \\
\hline RMG & & & & & & RMG \\
\hline
\end{tabular}

$\%$ of worms expressing NeuroPAL reporters

$$
1 L+2 R \quad 2 L+1 R \quad 2 L+2 R \quad L=R
$$

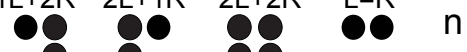

\begin{tabular}{|c|c|c|c|c|c|}
\hline & N2 & 0 & 0 & 10 & 0 \\
\hline & lin-32 & 23.3 & 30 & 3. & 43.4 \\
\hline & N2 & 0 & 0 & 10 & 0 \\
\hline & lin-32 & 6.7 & 3.3 & & 90 \\
\hline
\end{tabular}

Fig.8 
A

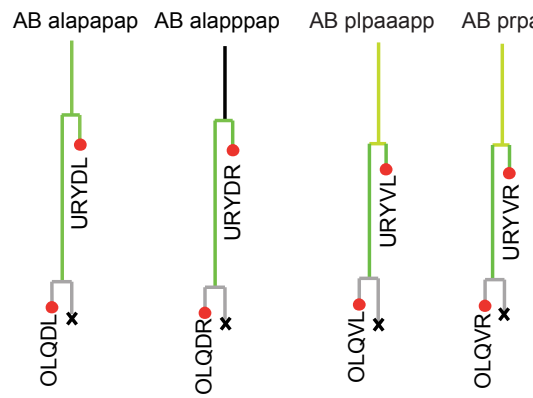

Dim expression not examined

Strong expression No expression

E \% of worms expressing OLQ NeuroPAL colors

$\stackrel{L}{\bigcirc} \quad 0<R \quad L=R$ no exp. $n$
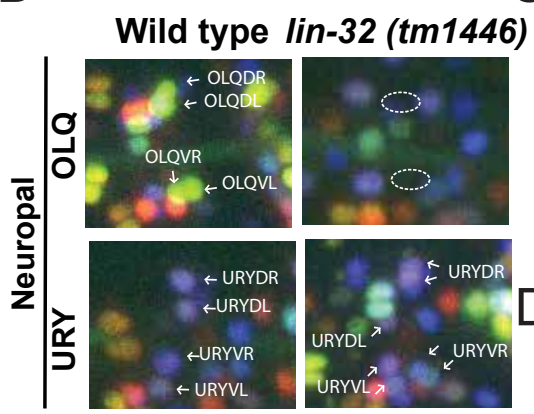

C
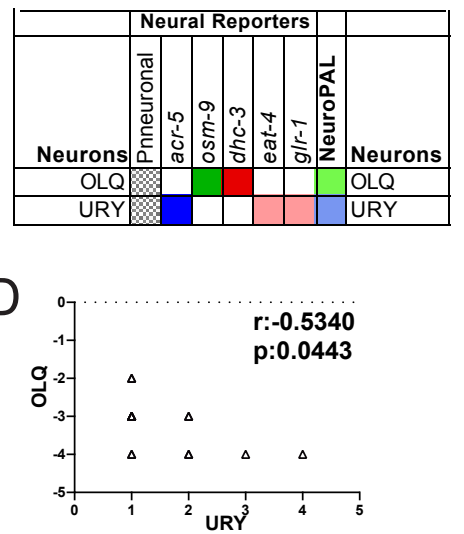

$\%$ of worms expressing URY NeuroPAL colors $1 L+2 R \quad 2 L+1 R \quad 2 L+2 R \quad L=R$ 0880080

\begin{tabular}{|c|c|c|c|c|c|c|}
\hline & N2 & 0 & 0 & 100 & 0 & 30 \\
\hline & lin-32 & 10 & 16.67 & 10 & 63.3 & 30 \\
\hline & N2 & 0 & 0 & 100 & 0 & 30 \\
\hline & lin-32 & 16.7 & 10 & 6.7 & 66.7 & 30 \\
\hline
\end{tabular}

$\%$ of worms with indicated ceh-32 pattern $1 L+2 R \quad 2 L+1 R \quad 2 L+2 R \quad L=R \quad n$

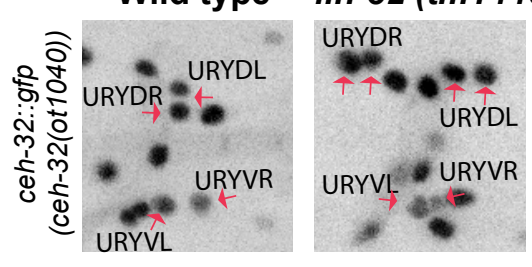

\begin{tabular}{|c|c|c|c|c|}
\hline N2 & 0 & 0 & 0 & 100 \\
\hline lin-32 & 26.1 & 42.8 & 5 & 26.1 \\
\hline N2 & 0 & 0 & 0 & 100 \\
\hline lin-32 & 26.1 & 13 & 18.1 & 42.8 \\
\hline
\end{tabular}

Fig.9 


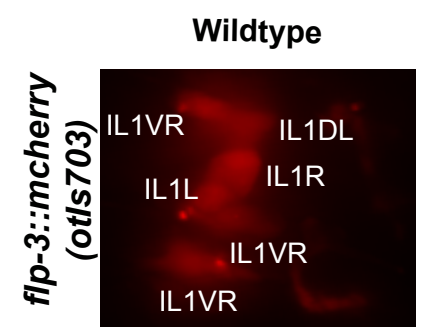

lin-32(tm1446)
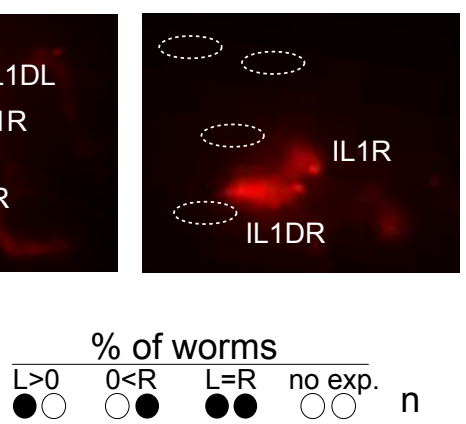

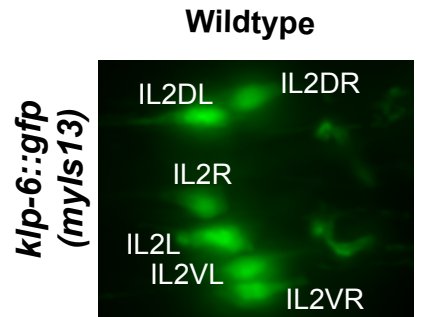

lin-32(tm1446)
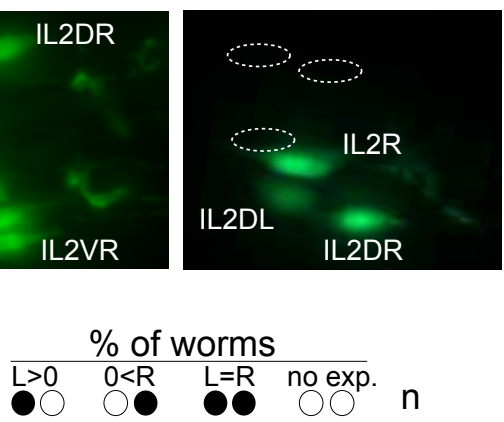

\begin{tabular}{|c|c|c|c|c|c|c|}
\hline $\begin{array}{l}\overline{\widetilde{W}} \\
\text { ஸे }\end{array}$ & N2 & 0 & 0 & 100 & 0 & 30 \\
\hline إٌ & lin-32 & 29.2 & 45.8 & 0 & 25 & 48 \\
\hline$\frac{\bar{T}}{0}$ & N2 & 0 & 0 & 100 & 0 & 30 \\
\hline آَّ & lin-32 & 12.5 & 29.2 & 0 & 45.8 & 48 \\
\hline 진| & N2 & 0 & 0 & 100 & 0 & 30 \\
\hline 딩 & lin-32 & 0 & 0 & 100 & 0 & 48 \\
\hline
\end{tabular}

Fig.S1 
